\title{
PACKING MEASURES AND DIMENSIONS ON CARTESIAN PRODUCTS
}

\author{
ONDŘEJ ZINDULKA
}

\begin{abstract}
Packing measures $\mathscr{P}^{g}(E)$ and Hewitt-Stromberg measures $\boldsymbol{\nu}^{g}(E)$ and their relatives are investigated. It is shown, for instance, that for any metric spaces $X, Y$ and any Hausdorff functions $f, g$

$$
\nu^{g}(X) \cdot \mathscr{P}^{h}(Y) \leqslant \mathscr{P}^{g h}(X \times Y)
$$

The inequality for the corresponding dimensions is established and used for a solution of a problem of $\mathrm{Hu}$ and Taylor: If $X \subseteq \mathbb{R}^{n}$, then

$$
\inf \left\{\overline{\operatorname{dim}}_{\mathrm{P}} X \times Y-\overline{\operatorname{dim}}_{\mathrm{P}} Y: Y \subseteq \mathbb{R}^{n}\right\}=\lim _{X_{n}} \inf _{X} \underline{\operatorname{dim}}_{\mathrm{B}} X_{n} .
$$

Corresponding dimension inequalities for products of measures are established.
\end{abstract}

\section{INTRODUCTION}

Consider separable metric spaces and their Hausdorff, packing and lower packing dimensions denoted, respectively, by $\operatorname{dim}_{H}, \overline{\operatorname{dim}}_{P}$ and $\underline{\operatorname{dim}}_{P}$ (the definitions are provided below). In 1981 Tricot [18] proved that if $X, Y \subseteq \mathbb{R}^{n}$, then

$$
\operatorname{dim}_{H} X+\overline{\operatorname{dim}}_{\mathrm{P}} Y \leqslant \overline{\operatorname{dim}}_{\mathrm{P}} X \times Y
$$

and this inequality was later generalized to arbitrary separable metric spaces by Howroyd [11. In $1993 \mathrm{Hu}$ and Taylor [2, (3.12)] asked if the inequality is sharp; in more detail, they defined, for $X \subseteq \mathbb{R}$, a dimension

$$
\operatorname{aDim} X=\inf \left\{\overline{\operatorname{dim}}_{\mathrm{P}} X \times Y-\overline{\operatorname{dim}}_{\mathrm{P}} Y: Y \subseteq \mathbb{R}\right\},
$$

noticed that (10) yields $\operatorname{dim}_{\mathrm{H}} X \leqslant \mathrm{aDim} X$ and asked if $\operatorname{aDim} X=\operatorname{dim}_{\mathrm{H}} X$ for all $X \subseteq \mathbb{R}$.

In 1996 two papers by Bishop and Peres 2 and Xiao 19 independently proved that if $X, Y \subseteq \mathbb{R}$ are compact, then the inequality (1) improves to

$$
\underline{\operatorname{dim}}_{\mathrm{P}} X+\overline{\operatorname{dim}}_{\mathrm{P}} Y \leqslant \overline{\operatorname{dim}}_{\mathrm{P}} X \times Y \text {, }
$$

doubting thus the conjectured $a \operatorname{Dim} X=\operatorname{dim}_{H} X$. Can one prove (3) for arbitrary $X, Y \subseteq \mathbb{R}$ or even in a more general setting? The proof in [2] is very technical and relies upon geometry of Euclidean spaces. On the other hand, the Xiao's [19] proof of (3) is a rather straightforward and simple application of Baire Category Theorem and can be thus easily extended to any compact metric spaces; and using the Joyce and Preiss theorem [12, to analytic metric spaces. But it seems impossible to

2010 Mathematics Subject Classification. 28A78, 28A80, 54E35.

Key words and phrases. Packing measure, lower packing measure packing dimension, lower packing dimension, cartesian product.

This project was supported by Department of Education of the Czech Republic, research project BA MSM 210000010. 
exploit the idea any further (actually [3] states (3) for arbitrary subsets of the line, but the proof therein is not very convincing).

It, however, turns out that a much finer, more general and sharper inequality can be proved in a rather general setting. Let us outline it in some detail. The dimensions in (3) are, like many other fractal dimensions, rarefaction indices of fractal measures: the packing dimension $\overline{\operatorname{dim}}_{\mathrm{P}} X$ is the number $s_{0}$ such that $\mathscr{P}^{s}(X)=0$ for all $s>s_{0}$ and $\mathscr{P}^{s}(X)=\infty$ for all $s<s_{0}$. The lower packing dimension is defined likewise from the so called Hewitt-Stromberg measures $\boldsymbol{\nu}^{s}(X)$. The inequality (3) is a trivial consequence of the integral inequality

$$
\mathscr{P}^{s+t}(E) \geqslant \int \boldsymbol{\nu}^{s}\left(E_{x}\right) \mathrm{d} \mathscr{P}^{t}(x)
$$

that holds for any subset $E \subseteq X \times Y$ of a product of metric spaces. This inequality, however, does not really help with the solution of the $\mathrm{Hu}$-Taylor problem. The crucial step towards its solution is the following observation. Given a set $E$ in a metric space and $s \geqslant 0$, define the lower box content $\boldsymbol{\nu}_{0}^{s}(E)=\liminf _{\delta \rightarrow 0} C_{\delta}(E) / \delta^{s}$, where $C_{\delta}(E)$ is the maximal number of points within $E$ that are mutually more than $\delta$ apart. The Hewitt-Stromberg measure $\boldsymbol{\nu}^{s}(E)$ obtains from $\boldsymbol{\nu}_{0}^{s}$ by the standard Method I construction: $\boldsymbol{\nu}^{s}(E)=\inf \sum_{n} \boldsymbol{\nu}_{0}^{s}\left(E_{n}\right)$, where the infimum is over all countable covers of $E$. The resulting set function is an outer Borel measure. Let's define another set function arising from $\boldsymbol{\nu}_{0}^{s}$ by the formula $\underline{\boldsymbol{\nu}}_{\rightarrow}^{s}(E)=$ $\inf \sup _{n} \boldsymbol{\nu}_{0}^{s}\left(E_{n}\right)$, where the infimum is this time over all increasing covers of $E$. If $\boldsymbol{\nu}_{0}^{s}$ were, like e.g. the upper box content, subadditive, we would get the same values as from Method I. But it is not. The set function $\underline{\nu}^{s}$ substantially differs from the Hewitt-Stromberg measure. It is a not measure, it is not even finitely subadditive, but it turns to be the right mean for solution of the $\mathrm{Hu}$-Taylor problem. Once one figures out the proof of (4), it is easy to improve it to

$$
\mathscr{P}^{s+t}(E) \geqslant \int \underline{\boldsymbol{\nu}}^{s}\left(E_{x}\right) \mathrm{d} \mathscr{P}^{t}(x) .
$$

Consequently the rarefaction index $\underset{\operatorname{dim}}{\longrightarrow}$ of $\underline{\boldsymbol{\nu}}^{s}$ satisfies, for any metric spaces $X, Y$,

$$
\underset{\mathrm{dim}}{\mathrm{P}} X+\overline{\operatorname{dim}}_{\mathrm{P}} Y \leqslant \overline{\operatorname{dim}}_{\mathrm{P}} X \times Y .
$$

Since $\operatorname{dim}_{P} X$ can be easily expressed in terms of lower box dimension (cf. Definition 4.1), it is not that esoteric. This improvement of inequality (3) gives the best-so-far lower estimate for the dimension of (2): aDim $X \geqslant \underset{\operatorname{dim}}{\longrightarrow} X$ for all $X \subseteq \mathbb{R}$ and actually for any metric space $X$.

As to the upper estimate of aDim, Xiao [19] proved that for any $X \subseteq \mathbb{R}, a \operatorname{Dim} X$ is estimated from above by the lower box dimension. And, luckily, analysis of his proof revealed that one can work upon its ideas to push the upper estimate down to $\underset{\mathrm{dim}}{\longrightarrow} X$. Therefore (6) is optimal. We arrived at the solution of Hu-Taylor problem:

Theorem. (i) $\operatorname{dim}_{\mathrm{P}} X+\overline{\operatorname{dim}}_{\mathrm{P}} Y \leqslant \overline{\operatorname{dim}}_{\mathrm{P}} X \times Y$ holds for any metric spaces $X, Y$.

(ii) For any $X \subseteq \mathbb{R}^{n}$ there is a compact set $Y \subseteq \mathbb{R}^{n}$ such that $\operatorname{dim}_{\mathrm{P}} X+\overline{\operatorname{dim}}_{\mathrm{P}} Y=$ $\overline{\operatorname{dim}}_{\mathrm{P}} X \times Y$.

(iii) In particular, $\inf \left\{\overline{\operatorname{dim}}_{\mathrm{P}} X \times Y-\overline{\operatorname{dim}}_{\mathrm{P}} Y: Y \subseteq \mathbb{R}^{n}\right\}=\operatorname{dim}_{\mathrm{P}} X$ for all $X \subseteq \mathbb{R}^{n}$.

Actually, with a proper extension of the definition of aDim, the theorem remains valid for any space $X$ whose Assouad dimension is finite. 
The paper is organized as follows. In Section 2 we recall in detail packing and Hewitt-Stromberg measure. Then we introduce scaled measures and upper/lower box and packing measures and list some elementary properties of these measures. In Section 3 we state and prove (5) and other integral inequalities involving these measures and derive inequalities for cartesian rectangles. In Section 4 the notions of upper/lower box and packing dimensions are recalled and the dimension ${\underset{\text { dim}}{P}}_{P}$ is introduced. Then we set up and prove (6) and other dimension inequalities following from the respective results of Section 3 . Section 5 is devoted to the solution of the $\mathrm{Hu}$-Taylor problem in a rather general setting. The paper is concluded with Section 6 that, besides various comments, presents applications to dimension theory of Borel measures, and lists some open problems.

\section{THE MEASURES}

In this section we set up definitions of packing and box measures whose behavior on cartesian products is investigated in the next section. We begin with recalling two common measures - the packing measure and the Hewitt-Stromberg measure. Then we generalize these notions, notice that via this generalization they are closely related and introduce the lower packing measure and a couple of more measures and pre-measures.

Since the technique used is rather standard, we present only few brief proofs.

Throughout the section, $X$ stands for a separable metric space with a metric $d$. Notation used includes $B(x, r)$ for the closed ball of radius $r>0$ centered at $x \in X ; \bar{A}$ for the closure of a set $A ;|A|$ for the cardinality of a set $A$; and $\omega$ for the set of all natural numbers including zero.

Pre-measures. It will be convenient to establish elementary features of the following constructions of pre-measures from pre-measures.

A set function is a mapping $\tau$ that assigns to each $E \subseteq X$ a value $\tau(E) \in[0, \infty]$. The notions of monotone, subadditive and countably subadditive set function are self-explaining. A set function will be called a pre-measure if it is monotone and $\tau(\emptyset)=0$. A pre-measure $\tau$ is metric if $\tau(A \cup B) \geqslant \tau(A)+\tau(B)$ whenever $A, B \subseteq X$ are separated, i.e. $\operatorname{dist}(A, B)>0$. Departing slightly from the common usage we call a pre-measure an outer measure if its restriction to the algebra of Borel sets is a Borel measure. An outer measure $\tau$ is Borel-regular if for each $A \subseteq X$ there is $B \supseteq A$ Borel with $\tau(B)=\tau(A)$.

The following is the Munroe's Method I construction, see [17]. Its point is that it produces a countably subadditive pre-measure from any pre-measure. Given a pre-measure $\tau$, the new pre-measure $\widehat{\tau}$ is defined by

$$
\widehat{\tau}(E)=\inf \left\{\sum_{n} \tau\left(E_{n}\right): E \subseteq \bigcup_{n} E_{n}\right\} .
$$

We shall also make use of a "directed" variation of Method $I$. Write $E_{n} \nearrow E$ to denote that $\left\langle E_{n}\right\rangle$ is an increasing sequence of sets with union $E$.

$$
\vec{\tau}(E)=\liminf _{E_{n} \nearrow E} \tau\left(E_{n}\right)=\inf \left\{\sup _{n} \tau\left(E_{n}\right): E_{n} \nearrow E\right\} .
$$

Let us call this construction Method $D$ for future reference. We list some elementary properties of the two operations.

Lemma 2.1. Let $\tau$ be a metric pre-measure on $X$.

(i) $\widehat{\tau}$ is an outer measure. 
(ii) If $\tau$ is Borel-regular, then so is $\widehat{\tau}$ and $\widehat{\tau} \leqslant \vec{\tau}$.

(iii) If $\tau$ is subadditive, then $\vec{\tau}=\widehat{\tau}$.

Proof. (i) By [17, Theorem 4], $\widehat{\tau}$ is countably subadditive. It is easy to check that since $\tau$ is metric, so is $\widehat{\tau}$. Hence (i) follows by [17, Theorem 19].

(ii) It is obvious that since $\tau$ is Borel-regular, so is $\widehat{\tau}$. It is also obvious that $\widehat{\tau} \leqslant \tau$; thus $\vec{\tau} \leqslant \vec{\tau}$. As $\widehat{\tau}$ is a Borel-regular outer measure, $\sup _{n} \widehat{\tau}\left(E_{n}\right)=\widehat{\tau}(E)$ holds for any sequence $E_{n} \nearrow E$, cf. [17, Theorem 4]. Hence $\widehat{\tau} \leqslant \vec{\tau}$ and $\widehat{\tau} \leqslant \vec{\tau}$ follows.

(iii) Let $E_{n} \nearrow E$. Set $A_{0}=E_{0}$ and $A_{n}=E_{n} \backslash E_{n-1}$ for $n>0$. Then $A_{n}$ 's cover $E$ and by assumption, $\sup _{n} \tau\left(E_{n}\right) \leqslant \sup _{n} \sum_{i \leqslant n} \tau\left(A_{i}\right)=\sum_{n \in \omega} \tau\left(A_{n}\right)$. This yields $\vec{\tau} \leqslant \widehat{\tau}$. The opposite inequality follows from (ii).

We now recall two classical measures: the packing measure and the HewittStromberg measure. They will play an important role in our considerations and moreover will motivate our definitions of scaled measures.

Packing measure. There are perhaps too many notions of packing and packing measure. We choose the one used e.g. in 11 and 12]; the other definitions are briefly discussed in Section 6. A family $\left\{\left(x_{i}, r_{i}\right): i \in I\right\} \subseteq X \times(0, \infty)$ is called a packing if $x_{i} \notin B\left(x_{j}, r_{j}\right)$ for all $i \neq j$ in $I$. Equivalently, if $d\left(x_{i}, x_{j}\right)>r_{i}$. It is a packing of a set $E \subseteq X$ if $x_{i} \in E$ for all $i \in I$. It is $\delta$-fine if $r_{i} \leqslant \delta$ for all $i \in I$.

We shall need the following simple lemma at a couple of instances.

Lemma 2.2. For any finite packing $\left\{\left(x_{i}, r_{i}\right): i \in I\right\}$ there is $\varepsilon>0$ such that $\left\{\left(x_{i}^{\prime}, r_{i}^{\prime}\right): i \in I\right\}$ is a packing whenever $d\left(x_{i}^{\prime}, x_{i}\right)<\varepsilon$ and $r_{i}^{\prime}<r_{i}+\varepsilon$ for all $i \in I$.

Proof. It is enough to put $\varepsilon=\frac{1}{3} \min \left\{d\left(x_{i}, x_{j}\right)-\max \left(r_{i}, r_{j}\right): i \neq j\right\}$.

Following [11], a Hausdorff function is a nondecreasing function $g:(0, \infty) \rightarrow$ $(0, \infty)$. No continuity of $g$ is a priori imposed. Hausdorff functions are (partially) ordered by $f \prec g$ iff $\overline{\lim }_{r \rightarrow 0} f(r) / g(r)=0$.

If $g$ is a Hausdorff function and $\pi=\left\{\left(x_{i}, r_{i}\right): i \in I\right\}$ a packing, we write

$$
g(\pi)=\sum\left\{g\left(r_{i}\right): i \in I\right\} .
$$

Definition 2.3 (11, 12]). Let $g$ be a Hausdorff function and $E \subseteq X$. Let

$$
\mathscr{P}_{0}^{g}(E)=\inf _{\delta>0} \mathscr{P}_{\delta}^{g}(E), \quad \text { where } \mathscr{P}_{\delta}^{g}=\sup \{g(\pi): \pi \text { is a } \delta \text {-fine packing of } E\} \text {. }
$$

The $g$-dimensional packing measure of $E$ is defined by $\mathscr{P}^{g}(E)=\widehat{\mathscr{P}_{0}^{g}}(E)$.

In the particular case when $g(r)=r^{s}$ for some constant $s \geqslant 0$, we write, as usual, $\mathscr{P}^{s}$ instead of $\mathscr{P}^{g}$; and the same license is used for other pre-measures and measures obtained from Hausdorff functions.

It is well-known and easy to see that $\mathscr{P}_{0}^{g}$ is an additive Borel-regular metric pre-measure and thus $\mathscr{P}^{g}$ is a Borel-regular outer measure.

Hewitt-Stromberg measure. For $F \subseteq X$ define gap $F=\inf \{d(x, y): x, y \in$ $F, x \neq y\}$. For $E \subseteq X$ and $\delta>0$ denote

$$
C_{\delta}(E)=\sup \{|F|: F \subseteq E, \operatorname{gap} F>\delta\}
$$

the $\delta$-capacity of $E$. The following natural notion appeared first in [10, (10.51)]. It was investigated and got the name in [8, 9. Another excellent reference is [7]. 
Definition 2.4 ([10]). Let $g$ be a Hausdorff function and $E \subseteq X$. Let

$$
\boldsymbol{\nu}_{0}^{g}(E)=\liminf _{\delta \rightarrow 0} C_{\delta}(E) g(\delta) .
$$

The $g$-dimensional Hewitt-Stromberg measure of $E$ is defined by $\boldsymbol{\nu}^{g}(E)=\widehat{\boldsymbol{\nu}_{0}^{g}}(E)$.

It is easy to check that $\boldsymbol{\nu}_{0}^{g}$ is a Borel-regular metric pre-measure (though is does not have to be subadditive) and thus $\boldsymbol{\nu}^{g}$ is a Borel-regular outer measure.

Scaled measures. A set $\Delta \subseteq(0, \infty)$ such that $0 \in \bar{\Delta}$ is termed a scale. We use $\Delta$ as a generic symbol for a scale. A packing $\left\{B\left(x_{i}, r_{i}\right): i \in I\right\}$ is $\Delta$-valued if $r_{i} \in \Delta$ for all $i \in I$. A $(\Delta, \delta)$-packing is a packing that is $\Delta \cap(0, \delta]$-valued, i.e. $\Delta$-valued and $\delta$-fine. A packing $\left\{B\left(x_{i}, r_{i}\right): i \in I\right\}$ is uniform if $r_{i}=r_{j}$ for all $i, j \in I$.

We now introduce an auxiliary notion of a $\Delta$-scaled packing measure. It is a straight generalization of the packing measure, the only difference is that the radii allowed in packings are limited to the set $\Delta$.

Definition 2.5. Let $g$ be a Hausdorff function, $\Delta$ a scale and $E \subseteq X$. Let $\mathscr{P}_{\Delta, 0}^{g}(E)=\inf _{\delta>0} \mathscr{P}_{\Delta, \delta}^{g}(E)$, where

$$
\mathscr{P}_{\Delta, \delta}^{g}(E)=\sup \{g(\pi): \pi \text { is a }(\Delta, \delta) \text {-packing of } E\} .
$$

The $g$-dimensional $\Delta$-packing measure of $E$ is defined by $\mathscr{P}_{\Delta}^{g}(E)=\widehat{\mathscr{P}_{\Delta, 0}^{g}}(E)$.

Let $\boldsymbol{\nu}_{\Delta, 0}^{g}(E)=\inf _{\delta>0} \boldsymbol{\nu}_{\Delta, \delta}^{g}(E)$, where

$$
\boldsymbol{\nu}_{\Delta, \delta}^{g}(E)=\sup \{g(\pi): \pi \text { is a uniform }(\Delta, \delta) \text {-packing of } E\} .
$$

The $g$-dimensional $\Delta$-box measure of $E$ is defined by $\boldsymbol{\nu}_{\Delta}^{g}(E)=\widehat{\boldsymbol{\nu}_{\Delta, 0}^{g}}(E)$.

Clearly $\boldsymbol{\nu}_{\Delta, 0}^{g} \leqslant \mathscr{P}_{\Delta, 0}^{g}$ and $\boldsymbol{\nu}_{\Delta}^{g} \leqslant \mathscr{P}_{\Delta}^{g}$. It is easy to check that the set function $\boldsymbol{\nu}_{\Delta, 0}^{g}$ can be equivalently defined in terms of capacity:

$$
\boldsymbol{\nu}_{\Delta, 0}^{g}(E)=\limsup _{\delta \in \Delta, \delta \rightarrow 0} C_{\delta}(E) \cdot g(\delta) .
$$

This equation shows the link to the Hewitt-Stromberg measure.

Here are some elementary facts about the scaled measures. (i) and (iv) are obvious, (ii) is a consequence of Lemma 2.2 and (iii) follows from Lemma 2.1

Lemma 2.6. (i) $\mathscr{P}_{\Delta, 0}^{g}$ and $\boldsymbol{\nu}_{\Delta, 0}^{g}$ are subadditive metric pre-measures,

(ii) $\mathscr{P}_{\Delta, 0}^{g}(E)=\mathscr{P}_{\Delta, 0}^{g}(\bar{E})$ for any set $E \subseteq X$, and likewise for $\boldsymbol{\nu}_{\Delta, 0}^{g}$.

(iii) $\mathscr{P}_{\Delta}^{g}$ and $\boldsymbol{\nu}_{\Delta}^{g}$ are Borel regular outer measures.

Upper measures. We now define upper packing and box measures as extreme cases of corresponding scaled measures. Among all scales, $(0, \infty)$ is the largest one. The corresponding scaled measures are thus largest among all scaled measures.

Definition 2.7. Let $g$ be a Hausdorff function and $E \subseteq X$. Let

$$
\begin{gathered}
\overline{\mathscr{P}}_{0}^{g}(E)=\sup _{\Delta} \mathscr{P}_{\Delta, 0}^{g}(E)=\mathscr{P}_{(0, \infty)}^{g}(E), \\
\overline{\boldsymbol{\nu}}_{0}^{g}(E)=\sup _{\Delta} \boldsymbol{\nu}_{\Delta, 0}^{g}(E)=\boldsymbol{\nu}_{(0, \infty)}^{g}(E) .
\end{gathered}
$$

The $g$-dimensional upper packing and box measures of $E$ are defined, respectively, by $\overline{\mathscr{P}}^{g}(E)=\widehat{\overline{\mathscr{P}}_{0}^{g}}(E)$ and $\overline{\boldsymbol{\nu}}^{g}(E)=\widehat{\overline{\boldsymbol{\nu}}_{0}^{g}}(E)$. 
It is clear that the upper packing measure $\overline{\mathscr{P}}^{g}$ is nothing but the packing measure $\mathscr{P}^{g}$ as defined in 2.3. We defined it just to point out the duality of (upper) packing measure and lower packing measure defined below. We prefer notation $\overline{\mathscr{P}}^{g}$ to make clear distinction between the upper and lower packing measures. As to $\overline{\boldsymbol{\nu}}_{0}^{g}$, it follows from (9) that

$$
\overline{\boldsymbol{\nu}}_{0}^{g}(E)=\limsup _{\delta \rightarrow 0} C_{\delta}(E) g(\delta)
$$

and thus $\overline{\boldsymbol{\nu}}^{g}$ is via (8) dual to the Hewitt-Stromberg measure. The upper box measures $\overline{\boldsymbol{\nu}}^{s}$ appear in many papers and books, explicitly e.g. in [16] and implicitly e.g. in [15, 5.3].

Note that $\overline{\mathscr{P}}^{g}$ and $\overline{\boldsymbol{\nu}}^{g}$ and the underlying pre-measures satisfy Lemma 2.6

Lower measures. Likewise we define lower packing and box measures as the lower extreme cases of corresponding scaled measures. The situation is more delicate, since there is no minimal scale.

Definition 2.8. Let $g$ be a Hausdorff function and $E \subseteq X$. Let

$$
\underline{\mathscr{P}}_{0}^{g}(E)=\inf _{\Delta} \mathscr{P}_{\Delta, 0}^{g}(E), \quad \underline{\boldsymbol{\nu}}_{0}^{g}(E)=\inf _{\Delta} \boldsymbol{\nu}_{\Delta, 0}^{g}(E),
$$

the infima over all scales. The $g$-dimensional lower packing and box measures of $E$ are defined, respectively, by $\underline{\boldsymbol{\nu}}^{g}(E)=\widehat{\boldsymbol{\nu}_{0}^{g}}(E)$ and $\overline{\boldsymbol{\nu}}^{g}(E)=\widehat{\boldsymbol{\nu}_{0}^{g}}(E)$.

Since the upper pre-measures $\overline{\mathscr{P}}_{0}^{g}$ and $\overline{\boldsymbol{\nu}}_{0}^{g}$ are subadditive, Method $D$ yields the same measures as Method I. It, however, is not the case of lower measures. That is why we also define $\underline{\mathscr{P}}^{g}(E)=\underline{\mathscr{P}}_{0}^{\vec{g}}(E)$ and $\underline{\boldsymbol{\nu}}^{g}(E)=\underline{\boldsymbol{\nu}}_{0}^{g}(E)$.

It follows from (9) that $\underline{\boldsymbol{\nu}}_{0}^{g}(E)=\liminf _{\delta \rightarrow 0} C_{\delta}(E) g(\delta)$. Thus $\underline{\boldsymbol{\nu}}_{0}^{g}=\boldsymbol{\nu}_{0}^{g}$ and $\underline{\boldsymbol{\nu}}^{g}=\boldsymbol{\nu}^{g}$, i.e. the lower box measure is just another name for the Hewitt-Stromberg measure. The lower packing measure and the two directed pre-measures seem to be new concepts.

Lemma 2.9. (i) $\underline{\mathscr{P}}_{0}^{g}$ and $\underline{\boldsymbol{\nu}}_{0}^{g}$ are metric pre-measures,

(ii) $\mathscr{P}_{0}^{g}(E)=\mathscr{\mathscr { P }}_{0}^{g}(\overline{\bar{E}})$ for any set $E \subseteq X$, and likewise for $\underline{\boldsymbol{\nu}}_{0}^{g}$,

(iii) $\underline{\mathscr{P}}^{g}$ and $\underline{\boldsymbol{\nu}}^{g}$ are Borel-regular outer measures,

(iv) if $\underline{\mathscr{P}}_{0}^{g}(E)<\infty$, then $E$ is totally bounded, and likewise for $\underline{\boldsymbol{\nu}}_{0}^{g}$.

Proof. (i) is straightforward, (ii) follows from Lemma 2.6(ii) and (iii) is a consequence of (ii). To prove (iv) it is enough to notice that if $\underline{\boldsymbol{\nu}}_{0}^{g}(E)<\infty$, then by (8) $C_{\delta}(E)<\infty$ for all $\delta>0$.

Lemma 2.10. For any Hausdorff function $g$

(i) $\mathscr{P}_{0}^{g}(E)=\inf _{\Delta} \sup \{g(\pi): \pi$ is a $\Delta$-valued packing of $E\}$,

(ii) $\underline{\boldsymbol{\nu}}_{0}^{g}(E)=\inf _{\Delta} \sup \{g(\pi): \pi$ is a $\Delta$-valued uniform packing of $E\}$,

(iii) $\mathscr{P}^{g} \leqslant \inf _{\Delta} \mathscr{P}_{\Delta}^{g}$,

(iv) $\underline{\boldsymbol{\nu}}^{g}=\inf _{\Delta} \boldsymbol{\nu}_{\Delta}^{g}$.

Proof. (i) For each scale $\Delta$ denote $S_{\Delta}=\sup \{g(\pi): \pi$ is a $\Delta$-valued packing of $E\}$ and $S=\inf _{\Delta} S_{\Delta}$. Note that if $\Delta$ is a scale and $\delta>0$, then $\Delta \cap(0, \delta)$ is also a scale. By the definition, $\mathscr{P}_{\Delta, 0}^{g}(E)=\inf _{\delta>0} S_{\Delta \cap(0, \delta)} \geqslant S$, which in turn yields $\underline{P}_{0}^{g}(E) \geqslant S$. The reverse inequality is obvious. (ii) is proved in the same manner. 
(iii) Clearly $\underline{\mathscr{P}}_{0}^{g} \leqslant \mathscr{P}_{\Delta, 0}^{g}$. Hence $\stackrel{\mathscr{P}}{\rightarrow}_{\rightarrow} \leqslant \overrightarrow{\mathscr{P}}_{\Delta, 0}^{g}$ and since Lemma 2.1(iii) yields $\overrightarrow{\mathscr{P}_{\Delta, 0}^{g}}=\mathscr{P}_{\Delta}^{g}$, we are done.

(iv) $\underline{\nu}_{\rightarrow}^{g} \leqslant \inf _{\Delta} \boldsymbol{\nu}_{\Delta}^{g}$ is proved the same way as (iii). To prove the reverse inequality, let $E \subseteq X$ and $s>\underline{\boldsymbol{\nu}}_{\rightarrow}^{g}(E)$. There is $E_{n} \nearrow E$ such that $\underline{\boldsymbol{\nu}}_{0}^{g}\left(E_{n}\right)<s$ for all $n$, i.e. there are scales $\Delta_{n}$ such that $C_{E_{n}}(r) g(r)<r$ for all $n$ and $r \in \Delta_{n}$. Choose $r_{n} \in \Delta_{n}$ so that the resulting sequence decreases to zero and let $\Delta=\left\{r_{n}: n \in \omega\right\}$. Proving that $\boldsymbol{\nu}_{\Delta, 0}^{g}\left(E_{n}\right) \leqslant s$ for all $n$, and thus $\boldsymbol{\nu}_{\Delta}^{g}(E) \leqslant s$, is straightforward.

We do not know if the inequality (iii) can be reversed.

Comparison. The inequalities $\underline{\mathscr{P}}^{g} \leqslant \mathscr{\mathscr { P }}^{g} \leqslant \underline{\mathscr{P}}_{0}^{g}$ and $\underline{\boldsymbol{\nu}}^{g} \leqslant \underline{\boldsymbol{\nu}}^{g} \leqslant \underline{\boldsymbol{\nu}}_{0}^{g}$ are trivial. As follows from Example 4.2 none of these four inequalities can be reversed. It is also clear that $\underline{\boldsymbol{\nu}}_{0}^{g} \leqslant \underline{\mathscr{P}}_{0}^{g}, \underline{\boldsymbol{\nu}}^{g} \leqslant \underline{\mathscr{P}}^{g}$ and $\underline{\boldsymbol{\nu}}^{g} \leqslant \underline{\mathscr{P}}^{g}$, but we do not know if they can be reversed. We only know that $\underline{\boldsymbol{\nu}}_{0}^{g}$ and $\underline{\mathscr{P}}_{0}^{g}$ have the same null sets.

Proposition 2.11. For any set $E \subseteq X, \underline{\mathscr{P}}_{0}^{g}(E)=0$ if and only if $\underline{\boldsymbol{\nu}}_{0}^{g}(E)=0$.

Proof. The forward implication is obvious. To prove the backward one assume $\underline{\boldsymbol{\nu}}_{0}^{g}(E)=0$. Then there is a sequence $r_{n} \downarrow 0$ such that $C_{r_{n}}(E) g\left(r_{n}\right) \leqslant 2^{-n}$. For $m \in \omega$ define a scale $\Delta_{m}=\left\{r_{n}: n>m\right\}$. If $\pi$ is a $\Delta_{m}$-valued packing, then

$$
g(\pi)=\sum_{n>m} \sum\left\{g\left(r_{n}\right):\left(x, r_{n}\right) \in \pi\right\} \leqslant \sum_{n>m} C_{r_{n}}(E) g\left(r_{n}\right) \leqslant \sum_{n>m} 2^{-n}=2^{-m} .
$$

Therefore $\underline{\mathscr{P}}_{\Delta_{m}, 0}^{g}(E) \leqslant 2^{-m}$ and consequently $\underline{\mathscr{P}}_{0}^{g}(E) \leqslant \inf _{m \in \omega} 2^{-m}=0$.

This proposition is enough to show that the measures $\underline{\boldsymbol{\nu}}^{g}$ and $\underline{\mathscr{P}}^{g}$ are close to each other:

Proposition 2.12. The following are equivalent:

(i) there is a countable cover $\left\{E_{n}\right\}$ of $E$ such that $\underline{\nu}_{0}^{g}\left(E_{n}\right)=0$ for all $n$,

(ii) there is $h \prec g$ such that $\underline{\boldsymbol{\nu}}^{h}(E)=0$,

(iii) there is $h \prec g$ such that $\underline{\mathscr{P}}^{h}(E)=0$.

Proof. (i) $\Leftrightarrow$ (ii) is proved in [8, Prop. 6]. The proof therein can be easily adapted to show that, via Proposition 2.11, (i) $\Rightarrow$ (iii), and (iii) $\Rightarrow$ (ii) is obvious.

The directed pre-measures are also close:

Proposition 2.13. The following are equivalent:

(i) there is $E_{n} \nearrow E$ such that $\underline{\nu}_{0}^{g}\left(E_{n}\right)=0$ for all $n$,

(ii) there is $E_{n} \nearrow E$ and a sequence $r_{n} \downarrow 0$ such that $C_{r_{n}}\left(E_{n}\right) g\left(r_{n}\right) \rightarrow 0$,

(iii) there is $h \prec g$ such that $\underline{\boldsymbol{\nu}}_{\rightarrow}^{h}(E)=0$,

(iv) there is $h \prec g$ such that $\overrightarrow{\mathscr{P}}^{h}(E)=0$,

(v) there is $h \prec g$ and a scale $\Delta$ such that $\mathscr{P}_{\Delta}^{h}(E)=0$.

Proof. (i) $\Rightarrow$ (ii): By virtue of (9) there is, for each $n$, a sequence $r_{i}^{n} \downarrow 0$ such that $r_{n}^{n} \rightarrow 0$ and $\lim _{i \rightarrow \infty} C_{r_{i}^{n}}\left(E_{n}\right) g\left(r_{i}^{n}\right)=0$. It is enough set $r_{n}=r_{n}^{n}$.

(ii) $\Rightarrow(\mathrm{v})$ : Since $\lim _{n \rightarrow \infty} C_{r_{n}}\left(E_{n}\right) g\left(r_{n}\right)=0$, there is clearly $h \prec g$ such that $\lim _{n \rightarrow \infty} C_{r_{n}}\left(E_{n}\right) h\left(r_{n}\right)=0$. Letting $\Delta=\left\{r_{n}: n \in \omega\right\}$ we have $\mathscr{P}_{\Delta}^{h}(E)=0$.

(v) $\Rightarrow$ (iv) $\Rightarrow$ (iii) are obvious and (iii) $\Rightarrow$ (i) follows from 2.14(i) below.

Proposition 2.14. If $g \prec h$, then for any set $E \subseteq X$ 
(i) $\underline{\nu}_{0}^{g}(E)<\infty \Rightarrow \underline{\mathscr{P}}_{0}^{h}(E)=0$,

(ii) $\underline{\boldsymbol{\nu}}^{g}(E)<\infty \Rightarrow \mathscr{P}^{h}(E)=0$,

(iii) $\underline{\nu}^{g}(E)<\infty \Rightarrow \stackrel{\mathscr{P}}{\rightarrow}^{h}(E)=0$ and there is a scale $\Delta$ such that $\mathscr{P}_{\Delta}^{h}(E)=0$.

Proof. (i) Using (91) it is clear that if $g \prec h$, then $\underline{\boldsymbol{\nu}}_{0}^{g}(E)<\infty$ yields $\underline{\boldsymbol{\nu}}_{0}^{h}(E)=0$. Now use Proposition 2.11. (ii) is an obvious consequence of (i). (iii) Suppose $\underline{\boldsymbol{\nu}}^{g}(E)<\infty$. Then, by (i), condition (i) of the above proposition is satisfied. Hence also conditions 2.13 (iv) and (v) are satisfied, which is enough.

These three propositions show that $\underline{\boldsymbol{\nu}}^{g}$ and $\underline{\mathscr{P}}^{g}$, as well as $\underline{\boldsymbol{\nu}}^{g}$ and $\stackrel{\mathscr{P}}{\rightarrow}^{g}$, respectively, are in a sense very close, as contrasted by the corresponding upper measures: As to the comparison of $\overline{\boldsymbol{\nu}}^{g}$ and $\overline{\mathscr{P}}^{g}$, needless to say that $\overline{\boldsymbol{\nu}}^{g} \leqslant \overline{\mathscr{P}}^{g}$, but not much more can be said, except that if $0<s<t$ and $\overline{\boldsymbol{\nu}}^{s}(E)=0$, then $\overline{\mathscr{P}}^{t}(E)=0$. This fact can be extracted e.g. from the proof of [15, Theorem 5.11]. It, however, is not difficult to show by example that the upper measures fail statements analogical to Propositions 2.11, 2.12 and 2.14,

Lipschitz maps. All of the (pre)-measures under consideration behave under Lipschitz maps as expected. The simple proof of the following is omitted.

Lemma 2.15. Let $s \geqslant 0$ and let $f: X \rightarrow Y$ be a c-Lipschitz map. Then $\underline{\mathscr{P}}^{s}(f(X)) \leqslant c^{s} \underline{\mathscr{P}}^{s}(X)$ and likewise for $\underline{\boldsymbol{\nu}}_{0}^{s}, \overline{\boldsymbol{\nu}}_{0}^{s}, \underline{\mathscr{P}}_{0}^{s}, \overline{\mathscr{P}}_{0}^{s}, \underline{\boldsymbol{\nu}}^{s}, \underline{\boldsymbol{\nu}}^{s}, \overline{\boldsymbol{\nu}}^{s}, \underline{\mathscr{P}}^{s}$ and $\overline{\mathscr{P}}^{s}$.

\section{Packing measures on Cartesian products}

This section is devoted to investigation of integral and product inequalities involving packing and box measures. Fix two metric spaces $X, Y$ and provide their cartesian product $X \times Y$ with the maximum metric. For a set $E \subseteq X \times Y$ and $x \in X$, the cross section $\{y \in Y:(x, y) \in E\}$ is denoted $E_{x}$ or $(E)_{x}$. Fix also a scale $\Delta$ and two Hausdorff functions $g, h$.

Lemma 3.1. For any set $E \subseteq X \times Y$

$$
\mathscr{P}_{\Delta, 0}^{g h}(E) \geqslant \mathscr{P}_{\Delta}^{h}(X) \cdot \inf _{x \in X} \underline{\nu}_{0}^{g}\left(E_{x}\right) .
$$

Proof. Let $c<\inf _{x \in X} \underline{\boldsymbol{\nu}}_{0}^{g}\left(E_{x}\right)$. For each $x$ there is a number $n \in \omega$ such that $\underline{\boldsymbol{\nu}}_{\delta}^{g}\left(E_{x}\right)>c$ for all $\delta<\frac{1}{n}$. Setting

$$
B_{n}=\left\{x: \underline{\boldsymbol{\nu}}_{\delta}^{g}\left(E_{x}\right)>c \text { for all } \delta<\frac{1}{n}\right\}
$$

we thus have $B_{n} \nearrow X$. Let $d<\mathscr{P}_{\Delta}^{h}(X)$. Lemmas 2.6(iii) and 2.1(iii) yield $n$ such that $d<\mathscr{P}_{\Delta, 0}^{h}\left(B_{n}\right)$. Hence there is $\delta_{0}>0$ such that for all $\delta<\delta_{0}$ there is a $(\Delta, \delta)$ packing $\pi=\left\{\left(x_{i}, r_{i}\right): i \in I\right\}$ of $B_{n}$ such that $h(\pi)>d$. We may assume $\delta_{0}<\frac{1}{n}$. Thus for each $i \in I$ there is a uniform $\delta$-fine packing $\pi_{i}=\left\{\left(y_{i j}, r_{i}\right): i \in K_{i}\right\}$ of $E_{x_{i}}$ such that $g\left(\pi_{i}\right)=\left|K_{i}\right| \cdot g\left(r_{i}\right)>c$. The collection $\sigma=\left\{\left(\left(x_{i}, y_{i j}\right), r_{i}\right): i \in I, j \in K_{i}\right\}$ is thus a $(\Delta, \delta)$-packing of $A$ and

$$
g h(\sigma)=\sum_{i \in I} \sum_{j \in K_{i}} g\left(r_{i}\right) h\left(r_{i}\right)=\sum_{i \in I}\left|K_{i}\right| g\left(r_{i}\right) h\left(r_{i}\right)>c \sum_{i \in I} h\left(r_{i}\right)>c d .
$$

Therefore $\mathscr{P}_{\Delta, \delta}^{g h}(A)>c d$. As this holds for any $\delta<\delta_{0}$ and all $d<\mathscr{P}_{\Delta}^{h}(X)$ and $c<\inf _{x \in X} \underline{\boldsymbol{\nu}}_{0}^{g}\left(E_{x}\right)$, we are done. 
Lemma 3.2. If $E \subseteq X \times Y$ is compact, then the mapping $x \mapsto \underline{\boldsymbol{\nu}}_{0}^{g}\left(E_{x}\right)$ is Borel measurable and

$$
\mathscr{P}_{\Delta, 0}^{g h}(E) \geqslant \int \underline{\boldsymbol{\nu}}_{0}^{g}\left(E_{x}\right) \mathrm{d} \mathscr{P}_{\Delta}^{h}(x) .
$$

Proof. We first show that if $E$ is compact, then $x \mapsto \underline{\boldsymbol{\nu}}_{0}^{g}\left(E_{x}\right)$ is Borel measurable. It follows from Lemma 2.2 that

$$
\forall r>0 \exists \varepsilon>0 C_{r+\varepsilon}(E)=C_{r}(E) .
$$

Thus for $x \in X$ fixed, the mapping $\delta \mapsto C_{\delta}\left(E_{x}\right)$ is right-continuous. Therefore the mapping $\delta \mapsto C_{\delta}\left(E_{x}\right) \cdot g(\delta)$ is right-continuous at each point of (right-)continuity of $g$. So if $Q \subseteq(0, \infty)$ is a dense countable set and $D$ the set of points of discontinuity of $g$, then

$$
\underline{\boldsymbol{\nu}}_{0}^{g}\left(E_{x}\right)=\liminf _{\substack{\delta \rightarrow 0 \\ \delta \in Q \cup D}} C_{\delta}\left(E_{x}\right) \cdot g(\delta) .
$$

As $g$ is nondecreasing, the set $D$ is countable. Therefore $x \mapsto \underline{\boldsymbol{\nu}}_{0}^{g}\left(E_{x}\right)$ obtains from a countable family of mappings of the form

$$
x \mapsto C_{\delta}\left(E_{x}\right) \cdot g(\delta), \quad \delta \in Q \cup D .
$$

Borel measurability of $x \mapsto \underline{\boldsymbol{\nu}}_{0}^{g}\left(E_{x}\right)$ will thus follow if we show that each of these mappings is Borel measurable. To that end we prove that for any $\delta>0$ and each integer $n$ the set $L=\left\{x \in X: C_{\delta}\left(E_{x}\right) \geqslant n\right\}$ is Borel, which is enough, as $g(\delta)$ is constant and $C_{\delta}\left(E_{x}\right)$ is integer-valued. For each $\varepsilon>0$ set

$$
L(\varepsilon)=\left\{x: \text { there is }\left\{y_{1}, y_{2}, \ldots, y_{n}\right\} \subseteq E_{x}, \operatorname{gap}\left\{y_{1}, y_{2}, \ldots, y_{n}\right\} \geqslant \delta+\varepsilon\right\} .
$$

By (12), $L=\bigcup_{\varepsilon>0} L(\varepsilon)$. Each of the sets $L(\varepsilon)$ is closed: Let $x_{k} \rightarrow x$ be a sequence in $L(\varepsilon)$ and $\left\{y_{k 1}, y_{k 2}, \ldots, y_{k n}\right\} \subseteq E_{x_{k}}$ sets witnessing $x_{k} \in L(\varepsilon)$. Choosing a subsequence if necessary, for each $i \leqslant n$ the sequence $\left(x_{k}, y_{i k}\right)$ converges to a point $\left(x, y_{i}\right) \in E$; this follows from compactness of $E$. The set $\left\{y_{1}, y_{2}, \ldots, y_{n}\right\}$ obviously witnesses $x \in L(\varepsilon)$. So $L(\varepsilon)$ is closed and therefore $L=\bigcup_{m \in \omega} L(1 / m)$ is $F_{\sigma}$ and hence Borel.

The next goal is to derive (11) from Lemma 3.1. As $E$ is compact, replacing $X$ and $Y$ with projections of $E$ we may assume both $X, Y$ compact. Write $\mu=\mathscr{P}_{\Delta}^{h}$.

We need to show that $\mathscr{P}_{\Delta, 0}^{g h}(E) \geqslant \int s \mathrm{~d} \mu$ for each simple function $s \leqslant \underline{\nu}_{0}^{g}\left(E_{x}\right)$. Let $s=\sum_{i=1}^{m} c_{i} \chi_{A_{i}}$ be such a function, with $A_{i}$ 's disjoint Borel sets and $c_{i}$ 's positive. If there is $i$ such that $\mu\left(A_{i}\right)=\infty$, then $\mathscr{P}_{\Delta, 0}^{g h}(E) \geqslant c_{i} \mu\left(A_{i}\right)=\infty$ by Lemma 3.1 Otherwise $\mu\left(A_{i}\right)<\infty$ for all $i$ and thus $A_{i}$ 's may be approximated from within with compact sets, for $X$ is compact: For any $\varepsilon>0$ and each $i$ there is a compact set $K_{i} \subseteq A_{i}$ such that $\mu\left(K_{i}\right)>\mu\left(A_{i}\right)-\frac{\varepsilon}{m c_{i}}$. Therefore

$$
\int s \mathrm{~d} \mu=\sum_{i=1}^{m} c_{i} \mu\left(A_{i}\right) \leqslant \sum_{i=1}^{m} c_{i}\left(\mu\left(K_{i}\right)+\frac{\varepsilon}{m c_{i}}\right)=\varepsilon+\sum_{i=1}^{m} c_{i} \mu\left(K_{i}\right) .
$$

For each $i$ put $E_{i}=E \cap\left(K_{i} \times Y\right)$. Apply Lemma 3.1 to $E_{i}$ 's to get

$$
c_{i} \mu\left(K_{i}\right) \leqslant \mathscr{P}_{\Delta, 0}^{g h}\left(E_{i}\right), \quad i=1,2, \ldots, m .
$$


Thus $\int s \mathrm{~d} \mu \leqslant \varepsilon+\sum_{i=1}^{m} \mathscr{P}_{\Delta, 0}^{g h}\left(E_{i}\right)$. As $K_{i}$ 's are disjoint compacta, so are $E_{i}$ 's. Therefore $E_{i}$ 's, being disjoint, are separated and thus Lemma 2.6(i) yields

$$
\sum_{i=1}^{m} \mathscr{P}_{\Delta, 0}^{g h}\left(E_{i}\right)=\mathscr{P}_{\Delta, 0}^{g h}\left(\bigcup_{i=1}^{m} E_{i}\right) \leqslant \mathscr{P}_{\Delta, 0}^{g h}(E)
$$

Therefore $\int s \mathrm{~d} \mu \leqslant \varepsilon+\mathscr{P}_{\Delta, 0}^{g h}(E)$. Since $\varepsilon>0$ and $s \leqslant \underline{\boldsymbol{\nu}}_{0}^{g}\left(E_{x}\right)$ were arbitrary, (11) follows.

Since the mappings $x \mapsto \underline{\boldsymbol{\nu}}_{0}^{g}\left(E_{x}\right), x \mapsto \underline{\boldsymbol{\nu}}^{g}\left(E_{x}\right), x \mapsto \underline{\boldsymbol{\nu}}_{0}^{g}\left(E_{x}\right)$ etc. need not be Borel measurable, we set up the following theorems in terms of the upper integral

$$
\int^{*} f \mathrm{~d} \mu=\inf \left\{\int \phi \mathrm{d} \mu: \phi \geqslant f \text { Borel measurable }\right\} .
$$

Lemma 3.3. For any set $E \subseteq X \times Y$

$$
\mathscr{P}_{\Delta, 0}^{g h}(E) \geqslant \int^{*} \underline{\boldsymbol{\nu}}_{0}^{g}\left(E_{x}\right) \mathrm{d} \mathscr{P}_{\Delta}^{h}(x) .
$$

Proof. As all quantities are intrinsic properties of $E$, mutatis mutandis we may assume $X, Y$ be complete metric spaces. If $\mathscr{P}_{\Delta, 0}^{g h}(E)=\infty$, there is nothing to prove. If $\mathscr{P}_{\Delta, 0}^{g h}(E)<\infty$, then $E$ is by Lemma 2.9 (iv) totally bounded. Therefore its closure $\bar{E}$ is compact: for $X \times Y$ is complete. Hence Lemma 3.2 yields, with the aid of Lemma 2.6(ii),

$$
\mathscr{P}_{\Delta, 0}^{g h}(E)=\mathscr{P}_{\Delta, 0}^{g h}(\bar{E}) \geqslant \int \underline{\boldsymbol{\nu}}_{0}^{g}\left((\bar{E})_{x}\right) \mathrm{d} \mathscr{P}_{\Delta}^{h}(x) \geqslant \int^{*} \underline{\boldsymbol{\nu}}_{0}^{g}\left(E_{x}\right) \mathrm{d} \mathscr{P}_{\Delta}^{h}(x) .
$$

Theorem 3.4. Let $X, Y$ be metric spaces. For any set $E \subseteq X \times Y$

$$
\mathscr{P}_{\Delta}^{g h}(E) \geqslant \int^{*} \underline{\nu}^{g}\left(E_{x}\right) \mathrm{d} \mathscr{P}_{\Delta}^{h}(x) .
$$

Proof. Let $E_{n} \nearrow E$. By Lemma 3.3, $\mathscr{P}_{\Delta, 0}^{g h}\left(E_{n}\right) \geqslant \int^{*} \underline{\nu}_{0}^{g}\left(E_{n}\right)_{x} \mathrm{~d} \mathscr{P}_{\Delta}^{h}(x)$ for each $n$. Therefore Levi's monotone convergence theorem yields

$$
\sup _{n} \mathscr{P}_{\Delta, 0}^{g h}\left(E_{n}\right) \geqslant \int_{n}^{*} \sup _{n} \underline{\boldsymbol{\nu}}_{0}^{g}\left(E_{n}\right)_{x} \mathrm{~d} \mathscr{P}_{\Delta}^{h}(x) \geqslant \int^{*} \underline{\boldsymbol{\nu}}_{\rightarrow}^{g}(E)_{x} \mathrm{~d} \mathscr{P}_{\Delta}^{h}(x)
$$

because $\left(E_{n}\right)_{x} \nearrow E_{x}$ for all $x \in X$. Take the infimum over all sequences $E_{n} \nearrow E$ to get $\overrightarrow{\mathscr{P}_{\Delta, 0}^{g}}(E) \geqslant \int_{\underline{\nu}^{*}}^{g}(E)_{x} \mathrm{~d} \mathscr{P}_{\Delta}^{h}(x)$. By Lemmas [2.6(iii) and 2.1)(iii), $\overrightarrow{\mathscr{P}}_{\Delta, 0}^{g}(E)=$ $\mathscr{P}_{\Delta}^{g}(E)$.

The main theorem of this section follows.

Theorem 3.5. Let $X, Y$ be metric spaces. For any set $E \subseteq X \times Y$
(i) $\overline{\mathscr{P}}^{g h}(E) \geqslant \int^{*} \underline{\nu}^{g}\left(E_{x}\right) \mathrm{d} \overline{\mathscr{P}}^{h}(x)$,
(ii) $\underline{\mathscr{P}}^{g h}(E) \geqslant \int^{*} \underline{\boldsymbol{\nu}}^{g}\left(E_{x}\right) \mathrm{d} \underline{\mathscr{P}}^{h}(x)$,
(iii) $\stackrel{\mathscr{P}}{\rightarrow}^{g h}(E) \geqslant \int^{*} \underline{\nu}^{g}\left(E_{x}\right) \mathrm{d} \underline{\mathscr{P}}^{h}(x)$,
(iv) $\stackrel{\mathscr{P}}{\rightarrow}^{g h}(E) \geqslant \inf _{x \in X} \underline{\boldsymbol{\nu}}^{g}\left(E_{x}\right) \cdot \mathscr{P}_{\rightarrow}^{h}(X)$. 
Proof. (i) is a particular case of the above theorem with $\Delta=(0, \infty)$.

(ii): Let $E \subseteq \bigcup_{n} E_{n}$. Use Lemma 3.3 for each $n$ and take infima over all scales, first on the right and then on the left, to get $\underline{\mathscr{P}}_{0}^{g h}\left(E_{n}\right) \geqslant \int^{*} \underline{\boldsymbol{\nu}}_{0}^{g}\left(E_{n}\right)_{x} \mathrm{~d} \underline{\mathscr{P}}^{h}(x)$. Thus by Lebesgue Theorem

$$
\sum_{n} \underline{\mathscr{P}}_{0}^{g h}\left(E_{n}\right) \geqslant \int^{*} \sum_{n} \underline{\boldsymbol{\nu}}_{0}^{g}\left(E_{n}\right)_{x} \mathrm{~d} \underline{\mathscr{P}}^{h}(x) \geqslant \int^{*} \underline{\boldsymbol{\nu}}^{g}(E)_{x} \mathrm{~d} \underline{\mathscr{P}}^{h}(x) .
$$

Take the infimum over all sequences $E_{n}$ such that $E \subseteq \bigcup_{n} E_{n}$ to get the required inequality.

(iii): Let $E_{n} \nearrow E$. As above, $\underline{\mathscr{P}}_{0}^{g h}\left(E_{n}\right) \geqslant \int^{*} \underline{\nu}_{0}^{g}\left(E_{n}\right)_{x} \mathrm{~d} \underline{\mathscr{P}}^{h}(x)$. Now proceed as in the proof of Theorem 3.4, using Levi's monotone convergence theorem. (iv) can be proved in the same manner.

Letting $E=X \times Y$, we get the following estimates for cartesian rectangles. The last inequality follows by analysis of the proof of Lemma 3.1.

Corollary 3.6. For any metric spaces $X, Y$

(i) $\overline{\mathscr{P}}^{g h}(X \times Y) \geqslant \overline{\mathscr{P}}^{h}(X) \cdot \underline{\boldsymbol{\nu}}^{g}(Y)$,

(ii) $\stackrel{\mathscr{P}}{\rightarrow}^{g h}(X \times Y) \geqslant \stackrel{\mathscr{P}}{\rightarrow}^{h}(X) \cdot \underline{\boldsymbol{\nu}}^{g}(Y)$,

(iii) $\underline{\mathscr{P}}^{g h}(X \times Y) \geqslant \underline{\mathscr{P}}^{h}(X) \cdot \underline{\boldsymbol{\nu}}^{g}(Y)$,

(iv) $\underline{\mathscr{P}}_{0}^{g h}(X \times Y) \geqslant \underline{\mathscr{P}}_{0}^{h}(X) \cdot \underline{\boldsymbol{\nu}}_{0}^{g}(Y)$.

A number of consequences can be derived from these theorems. As a sample we prove an estimate of packing measure of a domain of a Lipschitz mapping, similar to $[15,7.7]$.

Corollary 3.7. Let $X, Y$ be metric spaces and $c \geqslant 1$. Let $f: X \rightarrow Y$ be a $c$-Lipschitz map. For any $s, t \geqslant 0$

$$
\begin{gathered}
\int_{\underline{\boldsymbol{\nu}}^{t}}^{*}\left(f^{-1}(y)\right) \mathrm{d} \overline{\mathscr{P}}^{s}(y) \leqslant c^{s+t} \overline{\mathscr{P}}^{s+t}(X), \\
\int^{*} \underline{\boldsymbol{\nu}}^{t}\left(f^{-1}(y)\right) \mathrm{d} \underline{\mathscr{P}}^{s}(y) \leqslant c^{s+t} \underline{\mathscr{P}}^{s+t}(X) .
\end{gathered}
$$

Proof. Let $E=\{(x, f(x)): x \in X\} \subseteq X \times Y$ be the graph of $f$. Switching the roles of $X$ and $Y$, Theorem 3.4yields $\int^{*} \underline{\nu}^{t}\left(f^{-1}(y)\right) \mathrm{d} \overline{\mathscr{P}}^{s}(y) \leqslant \overline{\mathscr{P}}^{s+t}(E)$. Since the mapping $x \mapsto(x, f(x))$ is $c$-Lipschitz, Lemma 2.15 yields $\overline{\mathscr{P}}^{s+t}(E) \leqslant c^{s+t} \overline{\mathscr{P}}^{s+t}(X)$. The second inequality is proved the same way.

Remark 3.8. All of the inequalities of this section remain true if all $\mathscr{P}$ 's are replaced with $\boldsymbol{\nu}$ 's, with the same proofs, one only has to use uniform packings in place of packings. In particular, Theorem 3.5 reads

Theorem 3.9. Let $X, Y$ be metric spaces. For any set $E \subseteq X \times Y$

(i) $\overline{\boldsymbol{\nu}}^{g h}(E) \geqslant \int_{\underline{\boldsymbol{\nu}}^{g}}^{\text {g }}\left(E_{x}\right) \mathrm{d} \overline{\boldsymbol{\nu}}^{h}(x)$,

(ii) $\underline{\boldsymbol{\nu}}^{g h}(E) \geqslant \int^{*} \underline{\boldsymbol{\nu}}^{g}\left(E_{x}\right) \mathrm{d} \underline{\boldsymbol{\nu}}^{h}(x)$,

(iii) $\underline{\boldsymbol{\nu}}^{g h}(E) \geqslant \int^{*} \underline{\boldsymbol{\nu}}^{g}\left(E_{x}\right) \mathrm{d} \underline{\boldsymbol{\nu}}^{h}(x)$,

(iv) $\underline{\boldsymbol{\nu}}_{\rightarrow}^{g h}(E) \geqslant \inf _{x \in X} \stackrel{\boldsymbol{\nu}}{\rightarrow}^{g}\left(E_{x}\right) \cdot \underline{\boldsymbol{\nu}}_{\rightarrow}^{h}(X)$. 


\section{PACKING DIMENSIONS ON CARTESIAN PRODUCTS}

In this section we interpret the inequalities of the previous section in terms of fractal dimensions. We first recall the dimensions and introduce a new one related to the pre-measures $\underline{\boldsymbol{\nu}}^{s}$ and $\underline{\mathscr{P}}^{s}$. General reference: [15].

Fix $E \subseteq X$. A family $\mathcal{C}$ of sets is a $\delta$-cover of $E$ if it covers $E$ and $\operatorname{diam} C \leqslant \delta$ for each $C \in \mathcal{C}$. In this section we shall make frequent use of the covering number function

$$
N_{\delta}(E)=\min \{|\mathcal{C}|: \mathcal{C} \text { is a } \delta \text {-cover of } E\}, \quad \delta>0 .
$$

The well-known lower and upper box dimensions, (also called box-counting or Minkowski) of a nonempty set $E \subseteq X$ are equivalently defined, respectively, by

$$
\begin{aligned}
& \underline{\operatorname{dim}}_{\mathrm{B}} E=\liminf _{\delta \rightarrow 0} \frac{\log N_{\delta}(E)}{|\log r|}=\liminf _{\delta \rightarrow 0} \frac{\log C_{\delta}(E)}{|\log r|}, \\
& \overline{\operatorname{dim}}_{\mathrm{B}} E=\limsup _{\delta \rightarrow 0} \frac{\log N_{\delta}(E)}{|\log r|}=\limsup _{\delta \rightarrow 0} \frac{\log C_{\delta}(E)}{|\log r|} .
\end{aligned}
$$

Since

$$
N_{2 \delta}(E) \leqslant C_{\delta}(E) \leqslant N_{\delta}(E)
$$

for any set $E$, the limits in these definitions indeed equal. The upper and lower packing dimensions are, respectively, defined by, cf. [15],

$$
\begin{aligned}
& \overline{\operatorname{dim}}_{\mathrm{P}} E=\inf \left\{\sup _{n} \overline{\operatorname{dim}}_{\mathrm{B}} E_{n}: E \subseteq \bigcup_{n} E_{n}\right\}, \\
& \underline{\operatorname{dim}}_{\mathrm{P}} E=\inf \left\{\sup _{n} \underline{\operatorname{dim}}_{\mathrm{B}} E_{n}: E \subseteq \bigcup_{n} E_{n}\right\} .
\end{aligned}
$$

It is easy to check that the upper packing dimension may be equivalently defined by $\overline{\operatorname{dim}}_{\mathrm{P}} E=\inf \left\{\sup _{n} \overline{\operatorname{dim}}_{\mathrm{B}} E_{n}: E_{n} \nearrow E\right\}$. However, this modification of lower packing dimension gives a rise to a new dimension:

Definition 4.1. $\underline{\operatorname{dim}}_{\mathrm{P}} E=\inf \left\{\sup _{n} \underline{\operatorname{dim}}_{\mathrm{B}} E_{n}: E_{n} \nearrow E\right\}$

It is clear that $\underline{\operatorname{dim}}_{\mathrm{P}} X \leqslant \underline{\operatorname{dim}}_{\mathrm{P}} X \leqslant \underline{\operatorname{dim}}_{\mathrm{B}} X$ for any set $X$. The following example shows that the three dimensions are distinct: There is a compact set $X \subseteq \mathbb{R}$ such that $\underline{\operatorname{dim}}_{\mathrm{P}} X<\underline{\operatorname{dim}}_{\mathrm{P}} X<\underline{\operatorname{dim}}_{\mathrm{B}} X$ :

Example 4.2. We will define three sets compact $K_{0}, K_{1}, E \subseteq \mathbb{R}$ such that

(i) $\underline{\operatorname{dim}}_{\mathrm{B}} K_{0}=\underline{\operatorname{dim}}_{\mathrm{B}} K_{1}=0$,

(ii) ${\underset{\operatorname{dim}}{\mathrm{P}}}_{\mathrm{B}} K_{0} \cup \bar{K}_{1}=\frac{1}{2}$,

(iii) $\underline{\operatorname{dim}}_{\mathrm{B}} E=1$,

(iv) $E$ is countable.

The required set is $X=K_{0} \cup K_{1} \cup E$. Indeed, (i) and (iv) imply $\underline{\operatorname{dim}}_{\mathrm{P}} X=0$, (ii) and (iv) imply $\underset{\mathrm{dim}}{\mathrm{dim}} X=\frac{1}{2}$ and (iii) implies $\underline{\operatorname{dim}}_{\mathrm{B}} X=1$.

To define the sets $K_{0}$ and $K_{1}$ consider the set $2^{\omega}$ of all binary sequences and also the corresponding tree $2^{<\omega}$ of finite binary sequences, and the canonical mapping of $2^{\omega}$ onto $[0,1]$ given by $\widehat{x}=\sum_{n \in \omega} 2^{-n-1} x(n)$. For $p \in 2^{<\omega}$ let $[p]=\left\{x \in 2^{\omega}: p \subseteq x\right\}$ be the cone determined by $p$. It is clear that $C_{p}=\{\widehat{x}: x \in[p]\}$ is a closed binary interval of length $2^{-|p|}$. 
Choose an infinite set $D \subseteq \omega$ such that

$$
\varliminf_{n \rightarrow \infty} \frac{|D \cap n|}{n}=0 \text { and } \varlimsup_{n \rightarrow \infty} \frac{|D \cap n|}{n}=1
$$

and set

$$
\begin{aligned}
& K_{0}=\{\widehat{x}: x(n)=0 \text { for all } n \in D\}, \\
& K_{1}=\{\widehat{x}: x(n)=0 \text { for all } n \notin D\} .
\end{aligned}
$$

Let $n \in \omega$. There are exactly $2^{|n \backslash D|}$ binary intervals of length $2^{-n}$ that meet $K_{0}$. Hence

$$
N_{2^{-n}}\left(K_{0}\right)=2^{|n \backslash D|}
$$

and likewise

$$
N_{2^{-n}}\left(K_{1}\right)=2^{|n \cap D|} .
$$

Therefore (16) yields

$$
\underline{\operatorname{dim}}_{\mathrm{B}} K_{0}=\varliminf_{n \rightarrow \infty} \frac{\log N_{2^{-n}}\left(K_{0}\right)}{\left|\log 2^{-n}\right|}=\varliminf_{n \rightarrow \infty} \frac{|n \backslash D|}{n}=1-\varlimsup_{n \rightarrow \infty} \frac{|n \cap D|}{n}=0
$$

and likewise

$$
\underline{\operatorname{dim}}_{\mathrm{B}} K_{1}=\varliminf_{n \rightarrow \infty} \frac{\log N_{2^{-n}}\left(K_{1}\right)}{\left|\log 2^{-n}\right|}=\varliminf_{n \rightarrow \infty} \frac{|n \cap D|}{n}=0 .
$$

Thus the sets $K_{0}, K_{1}$ satisfy (i). To show (ii), we first claim that there is an infinite set $F \subseteq \omega$ such that

$$
\frac{n-1}{2}<|n \cap D| \leqslant \frac{n}{2} .
$$

Indeed, (16) yields $n$ arbitrarily large such that $\frac{|D \cap n|}{n} \leqslant \frac{1}{2}$ but $\frac{|D \cap(n+1)|}{n+1}>\frac{1}{2}$. Hence $|D \cap(n+1)|=|D \cap n|+1$ and the two inequalities imply (19).

Using (17), (18) and (19) it follows that

$$
\begin{aligned}
\underline{\operatorname{dim}}_{\mathrm{B}} K_{0} \cup K_{1} & \leqslant \varliminf_{n \rightarrow \infty} \frac{\log \left(2^{|n \backslash D|}+2^{|n \backslash D|}\right)}{\left|\log 2^{-n}\right|} \\
& \leqslant \underline{\lim } \frac{\log \left(2^{(n+1) / 2}+2^{n / 2}\right)}{n} \leqslant \frac{\lim }{n \in F} \frac{\log 2^{1+(n+1) / 2}}{n}=\frac{1}{2}
\end{aligned}
$$

and in particular $\operatorname{dim}_{\mathrm{P}} K_{0} \cup K_{1} \leqslant \frac{1}{2}$.

To prove the opposite inequality suppose for contrary that $X_{n} \nearrow K_{0} \cup K_{1}$ are such that $\underline{\operatorname{dim}}_{\mathrm{B}} X_{n}<\frac{1}{2}$ for all $n$. With no harm done we may suppose $X_{n}$ 's closed. By the Baire category argument there is an open set $U$ that meets both $K_{0}$ and $K_{1}$ and $\underline{\operatorname{dim}}_{\mathrm{B}} U \cap\left(K_{0} \cup K_{1}\right)<\frac{1}{2}$. Suppose without loss of generality that $U=I_{0} \cup I_{1}$, where $I_{0}$ meets $K_{0}, I_{1}$ meets $K_{1}$ and $I_{0}$ and $I_{1}$ are non-overlapping binary intervals of the same length, say $2^{-m}$. If $n>m$, then the number of binary intervals of length $2^{-n}$ that meet $I_{0} \cap K_{0}\left(I_{1} \cap K_{1}\right.$, respectively) is exactly $2^{\left|A_{n}\right|}\left(2^{\left|B_{n}\right|}\right)$, where $A_{n}=\{i \in \omega \backslash D: m \leqslant i<n\}$ and $B_{n}=\{i \in \omega \cap D: m \leqslant i<n\}$. Since $\left|A_{n} \cup B_{n}\right|=n-m$, we have $\max \left(\left|A_{n}\right|,\left|B_{n}\right|\right) \geqslant \frac{n-m}{2}$. Thus $N_{2^{-n}}\left(\left(K_{0} \cup K_{1}\right) \cap U\right) \geqslant$ $2^{(n-m) / 2}$, which in turn yields $\underline{\operatorname{dim}}_{\mathrm{B}}\left(K_{0} \cup K_{1}\right) \cap U \geqslant \frac{1}{2}$ : the desired contradiction. We conclude that $\operatorname{dim}_{\mathrm{P}} K_{0} \cup K_{1} \geqslant \frac{1}{2}$. Thus (ii) holds.

Finally let $E=\left\{\frac{1}{\log n}: n \in \omega, n \geqslant 2\right\} \cup\{0\}$. Routine calculation proves that $\underline{\operatorname{dim}}_{\mathrm{B}} E=1$. Thus (iii) and (iv) hold. 
Let us now see how the dimensions we described are related to the measures and pre-measures defined in the previous section. It is easy to check that all of the measures $\overline{\mathscr{P}}^{s}, \overline{\boldsymbol{\nu}}^{s}, \underline{\mathscr{P}}^{s}, \underline{\boldsymbol{\nu}}^{s}$ and the pre-measures $\overline{\mathscr{P}}_{0}^{s}, \overline{\boldsymbol{\nu}}_{0}^{s}, \underline{\mathscr{P}}_{0}^{s}, \underline{\boldsymbol{\nu}}_{0}^{s}, \underline{\mathscr{P}}^{s}, \underline{\boldsymbol{\nu}}^{s}$ are "rarefaction indices": If $\mathscr{L}^{s}$ is any of them, then

$$
\inf \left\{s: \mathscr{L}^{s}(E)=0\right\}=\sup \left\{s: \mathscr{L}^{s}(E)=\infty\right\} \text {. }
$$

Each of these (pre)-measures is linked to one of the above fractal dimensions by a common pattern: Tricot [18] proved that $\overline{\operatorname{dim}}_{\mathrm{B}} E=\inf \left\{s: \overline{\mathscr{P}}_{0}^{s}(E)=0\right\}=\inf \{s:$ $\left.\overline{\boldsymbol{\nu}}_{0}^{s}(E)=0\right\}$ and also that $\overline{\operatorname{dim}}_{\mathrm{P}} E=\inf \left\{s: \overline{\mathscr{P}}^{s}(E)=0\right\}=\inf \left\{s: \overline{\boldsymbol{\nu}}^{s}(E)=0\right\}$. It is folklore (and very easy to prove) that $\underline{\operatorname{dim}}_{\mathrm{B}} E=\inf \left\{s: \underline{\boldsymbol{\nu}}_{0}^{s}(E)=0\right\}$ and $\underline{\operatorname{dim}}_{\mathrm{P}} E=\inf \left\{s: \underline{\boldsymbol{\nu}}^{s}(E)=0\right\}$, cf. e.g. [15. Combining with Propositions 2.11 2.14 yields a list of equivalent definitions of the dimensions under consideration.

Proposition 4.3. For any set $E \subseteq X$

(i) $\overline{\operatorname{dim}}_{\mathrm{B}} E=\inf \left\{s: \overline{\mathscr{P}}_{0}^{s}(E)=0\right\}=\inf \left\{s: \overline{\boldsymbol{\nu}}_{0}^{s}(E)=0\right\}$,

(ii) $\underline{\operatorname{dim}}_{\mathrm{B}} E=\inf \left\{s: \mathscr{P}_{0}^{s}(E)=0\right\}=\inf \left\{s: \underline{\boldsymbol{\nu}}_{0}^{s}(E)=0\right\}$,

(iii) $\overline{\operatorname{dim}}_{\mathrm{P}} E=\inf \left\{s: \overline{\mathscr{P}}^{s}(E)=0\right\}=\inf \left\{s: \overline{\boldsymbol{\nu}}^{s}(E)=0\right\}$,

(iv) $\underline{\operatorname{dim}}_{P} E=\inf \left\{s: \mathscr{P}^{s}(E)=0\right\}=\inf \left\{s: \underline{\boldsymbol{\nu}}^{s}(E)=0\right\}$,

(v) $\underline{\operatorname{dim}}_{P} E=\inf \left\{s: \mathscr{P}^{s}(E)=0\right\}=\inf \left\{s: \underline{\nu}^{s}(E)=0\right\}$

$=\inf \left\{s: \exists \Delta \mathscr{P}_{\Delta}^{s}(E)=0\right\}=\inf \left\{s: \exists \Delta \vec{\nu}_{\Delta}^{s}(E)=0\right\}$.

Straightforward application of these identities to Theorems 3.9, 3.5 and Corollary 3.6 yields the corresponding dimension inequalities:

Theorem 4.4. Let $X, Y$ be metric spaces and $E \subseteq X \times Y$. Let $A \subseteq X$ be a set such that $E_{x} \neq \emptyset$ for all $x \in A$. Then

(i) $\overline{\operatorname{dim}}_{\mathrm{P}} E \geqslant \overline{\operatorname{dim}}_{\mathrm{P}} A+\inf _{x \in A} \stackrel{\operatorname{dim}}{\mathrm{P}} E_{x}$,

(ii) $\underline{\operatorname{dim}}_{\mathrm{P}} E \geqslant \underline{\operatorname{dim}}_{\mathrm{P}} A+\inf _{x \in A} \underline{\operatorname{dim}}_{\mathrm{P}} E_{x}$,

(iii) $\underset{\operatorname{dim}_{\mathrm{P}}}{ } E \geqslant{\underset{\operatorname{dim}}{\longrightarrow}}_{\mathrm{d}} A+\inf _{x \in A} \stackrel{\operatorname{dim}_{\mathrm{P}}}{\longrightarrow} E_{x}$.

Corollary 4.5. For any metric spaces $X, Y$

(i) $\overline{\operatorname{dim}}_{\mathrm{P}} X \times Y \geqslant \overline{\operatorname{dim}}_{\mathrm{P}} X+{\underset{\operatorname{dim}}{\mathrm{P}}}_{\mathrm{P}} Y$,

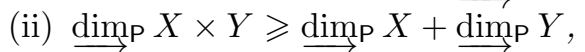

(iii) $\underline{\operatorname{dim}}_{\mathrm{P}} X \times Y \geqslant \underline{\operatorname{dim}}_{\mathrm{P}} X+\underline{\operatorname{dim}}_{\mathrm{P}} Y$.

\section{Solution of the Hu-Taylor problem}

The $\mathrm{Hu}$ and Taylor [2] definition of aDim (cf. (2) ) trivially extends to subsets of Euclidean spaces: for $X \subseteq \mathbb{R}^{m}$ let

$$
\operatorname{aDim} X=\min \left\{\overline{\operatorname{dim}}_{\mathrm{P}} X \times Z-\overline{\operatorname{dim}}_{\mathrm{P}} Z: Z \subseteq \mathbb{R}^{m}\right\}
$$

We employ the idea of Xiao [19] to show that $\operatorname{aDim} X=\operatorname{dim}_{\mathrm{P}} X$ for any $X \subseteq \mathbb{R}^{m}$ and actually for any metric space of finite Assouad dimension.

We make heavy use of the capacity and covering number functions introduced in (77) and (14). The following elementary estimates will be needed. If $X, Y$ are metric spaces and $r>0$, then

$$
\begin{aligned}
& C_{r}(X \times Y) \leqslant N_{r}(X) C_{r}(Y), \\
& N_{r}(X \times Y) \leqslant N_{r}(X) N_{r}(Y) .
\end{aligned}
$$


Let us recall the notion of Assouad dimension and related material. The interested reader is referred to J. Luukkainen's paper [14. Given $Q \geqslant 0$ and $m \geqslant 0$, a metric space $(X, d)$ is termed $(Q, m)$-homogeneous if $|A| \leqslant Q(b / a)^{m}$ whenever $a>0$ and $b \geqslant a$ are numbers and $A \subseteq X$ a set with $a \leqslant d(x, y) \leqslant b$ if $x, y \in A$ and $x \neq y$. It is easy to check that $X$ is $(Q, m)$-homogeneous if and only if $C_{r}(E) \leqslant Q\left(\frac{\operatorname{diam} E}{r}\right)^{m}$ for every set $E \subseteq X$ and every $r \leqslant \operatorname{diam} E$, and that is the definition we shall use.

The space $X$ is termed $m$-homogeneous if it is $(Q, m)$-homogeneous for some $Q$; and $X$ is termed countably $(Q, m)$-homogeneous if it is a countable union of $(Q, m)$ homogeneous subspaces, and likewise countably $m$-homogeneous if it is a countable union of $m$-homogeneous subspaces.

P. Assouad [1 defined what is now called Assouad dimension: If $X$ is a metric space, then

$$
\operatorname{dim}_{\mathrm{A}} X=\inf \{m>0: X \text { is } m \text {-homogeneous }\} .
$$

We also introduce the countably stable modification of $\operatorname{dim}_{\mathrm{A}} X$ :

$$
\operatorname{dim}_{\sigma \mathrm{A}} X=\inf \left\{\sup _{i} \operatorname{dim}_{\mathrm{A}} X_{i}:\left\{X_{i}\right\} \text { is a countable cover of } X\right\} .
$$

Spaces of finite Assouad dimension are also called $\beta$-spaces or doubling spaces and various other names and similar concepts are in use, e.g. D. G. Larman's 13. J. Luukkainen's paper [14] is a good source of information including an ample list of references.

We shall need the following simple lemma.

Lemma 5.1. Let $X$ be a $(Q, m)$-homogeneous metric space.

(i) If $0<r<t$, then $C_{r}(X) r^{m} \leqslant 2^{m} Q C_{t}(X) t^{m}$,

(ii) $\overline{\boldsymbol{\nu}}_{0}^{m}(X) \leqslant 2^{m} Q \underline{\boldsymbol{\nu}}_{0}^{m}(X)$.

Proof. (i) Suppose $C_{t}(X)<\infty$ and let $E \subseteq X$ be a maximal set with gap $E>t$. Then the family of balls $\{B(x, t): x \in E\}$ covers $X$. Therefore

$$
C_{r}(X) \leqslant \sum_{x \in E} C_{r}(B(x, r)) \leqslant|E| Q\left(\frac{2 t}{r}\right)^{m} \leqslant C_{t}(X) Q 2^{m}\left(\frac{t}{r}\right)^{m} .
$$

(ii) Let $r_{n} \downarrow 0$ be such that $\lim C_{r_{n}}(X) r_{n}^{m}=\underline{\boldsymbol{\nu}}_{0}^{m}(X)$. If $r_{n+1} \leqslant r \leqslant r_{n}$, then (i) yields $C_{r}(X) r^{m} \leqslant 2^{m} Q C_{r}(X) r_{n}^{m}$ and (ii) follows on letting $n \rightarrow \infty$.

Theorem 5.2. Let $\Delta$ be a scale and $0 \leqslant s \leqslant m \in \omega$. There is a compact set $\mathbf{Z} \subseteq \mathbb{R}^{m}$ such that $\overline{\boldsymbol{\nu}}^{m-s}(\mathbf{Z})=\overline{\boldsymbol{\nu}}_{0}^{m-s}(\mathbf{Z})=1$ and

(i) $\overline{\boldsymbol{\nu}}_{0}^{m}(X \times \mathbf{Z}) \leqslant 2^{m} Q \boldsymbol{\nu}_{\Delta, 0}^{s}(X)$ for every $(Q, m)$-homogeneous space $X$,

(ii) $\overline{\boldsymbol{\nu}}^{m}(X \times Z) \leqslant 2^{m} Q \boldsymbol{\nu}_{\Delta}^{s}(X)$ for every countably $(Q, m)$-homogeneous space $X$.

Proof. We prove only statement (i), as (ii) is its trivial consequence. If $s=0$, put $\mathrm{Z}=[0,1]^{m}$. In this case the inequality reduces to $\overline{\boldsymbol{\nu}}_{0}^{m}\left(X \times[0,1]^{m}\right) \leqslant 2^{m} Q|X|$, which is trivially satisfied for $X$ both finite or infinite.

If $s=m>0$, put $\mathbf{Z}=\{0\}$. In this case the inequality reduces to $\overline{\boldsymbol{\nu}}_{0}^{m}(X) \leqslant$ $2^{m} Q \boldsymbol{\nu}_{\Delta, 0}^{m}(X)$, which is nothing but Lemma 5.1. We will thus suppose that $0<s<$ $m$ and let $p=\frac{s}{m}$ throughout the proof.

We first construct recursively a decreasing sequence $r_{n} \rightarrow 0$ in $\Delta$ and an integervalued sequence $g \in \omega^{\omega}$ such that, letting

$$
\begin{aligned}
G(n) & =g(0) g(1) \ldots g(n-1), \\
u_{n} & =g(n) r_{n+1}
\end{aligned}
$$


we have, for all $n$,

$$
\begin{aligned}
1-\frac{1}{n} & <G(n) r_{n}^{1-p}<1+\frac{1}{n}, \\
r_{n+1} & <u_{n}<\frac{r_{n}}{n} .
\end{aligned}
$$

Let $G(0)=1$, choose any $r_{0} \in \Delta$ and recursively choose $r_{n+1} \in \Delta$ small enough so that $r_{n+1}^{p}<r_{n}$ and the interval $\left(\frac{1-\frac{1}{n+1}}{G(n) r_{n+1}^{1-p}}, \frac{1+\frac{1}{n+1}}{G(n) r_{n+1}^{1-p}}\right)$ is long enough to contain an even integer. Let it be $g(n)$. Thus

$$
\frac{1-\frac{1}{n+1}}{G(n) r_{n+1}^{1-p}}<g(n)<\frac{1+\frac{1}{n+1}}{G(n) r_{n+1}^{1-p}}
$$

and therefore $1-\frac{1}{n+1}<G(n+1) r_{n+1}^{1-p}<1+\frac{1}{n+1}$, as required. Since $g(n) \geqslant 2$, we also have $r_{n+1}<u_{n}$. Condition $r_{n+1}^{p}<r_{n}$ in conjunction with (24) ensures $u_{n}<r_{n} / n$.

Next we define the space $Z$. Let

$$
\begin{aligned}
& \mathrm{T}=\left\{x \in \omega^{\omega}: \forall n x(n)<g(n)\right\}, \\
& \mathrm{T}^{\bullet}=\left\{\tau \in \omega^{<\omega}: \exists x \in \mathrm{T} \tau \subseteq x\right\}, \quad \mathrm{T}_{n}^{\bullet}=\left\{\tau \in \mathrm{T}^{\bullet}:|\tau|=n\right\} .
\end{aligned}
$$

For each $\tau \in \mathrm{T}^{\bullet}$ define intervals $I_{\tau}=\left[a_{\tau}, b_{\tau}\right]$ recursively as follows. $I_{\emptyset}=\left[0, r_{0}\right]$. Now suppose $n \in \omega, \tau \in \mathrm{T}_{n}^{\bullet}$ and $I_{\tau}=\left[a_{\tau}, b_{\tau}\right]$ is defined. For $i<g(n)$ let $a_{\tau i}=a_{\tau}+i r_{n+1}$, $b_{\tau i}=a_{\tau i}+r_{n+1}=a_{\tau}+(i+1) r_{n+1}$. It is clear that, for any $\tau$ of length $n$, the family $\left\{I_{\tau i}: i<g(n)\right\}$ consists of adjacent non-overlapping equally sized intervals of length $r_{n+1}$ and that its union is an interval of length $u_{n}$; and since $u_{n}<r_{n}$, the union is contained in $I_{\tau}$. Set

$$
\mathrm{K}=\bigcap_{n \in \omega} \bigcup\left\{I_{\tau}: \tau \in \mathrm{T}_{n}^{\bullet}\right\}, \quad \mathrm{Z}=\mathrm{K}^{m} .
$$

In order to show that $\overline{\boldsymbol{\nu}}^{m-s}(Z)=\overline{\boldsymbol{\nu}}_{0}^{m-s}(Z)=1$ it is enough to establish the following claim. Let $\mu$ be the evenly distributed Borel probability measure on Z. In more detail, $\mu$ is the cartesian power of the Borel measure $\lambda$ on $\mathrm{K}$ that is determined by its values on $I_{\tau}$ 's: if $\tau \in \mathrm{T}_{n}^{\bullet}$, then $\lambda\left(I_{\tau}\right)=1 / G(n)$.

Lemma 5.3. $\mu(E)=\overline{\boldsymbol{\nu}}^{m-s}(E)$ for every Borel set $E \subseteq \mathrm{Z}$ and $\overline{\boldsymbol{\nu}}_{0}^{m-s}(\mathrm{Z})=1$.

Proof. For each $n \in \omega$ and every $\boldsymbol{\tau}=\left\langle\tau_{i}: i<m\right\rangle \in\left(\mathbf{T}_{n}^{\bullet}\right)^{m}$ define $J_{\boldsymbol{\tau}}=$ $\prod_{i<m}\left[a_{\tau_{i}}, a_{\tau_{i}+u_{n}}\right)$. Let $B \subseteq \mathrm{K}$ be Borel. Consider the set $S=\left\{\boldsymbol{\tau} \in\left(\mathrm{T}_{n}^{\bullet}\right)^{m}\right.$ : $\left.J_{\boldsymbol{\tau}} \cap B \neq \emptyset\right\}$. Pick one point of $B$ in every $J_{\boldsymbol{\tau}}, \boldsymbol{\tau} \in S$. Thus chosen points are mutually more than $r_{n}-u_{n}$ apart and thus witness $C_{r_{n}-u_{n}}(B) \geqslant|S|$. On the other hand, $\mu(B) \leqslant \sum_{I \in S} \mu(I)=|S| \cdot \frac{1}{G(n)^{m}}$. Therefore

$$
\mu(B) \leqslant \frac{|S|}{G(n)^{m}} \leqslant \frac{C_{r_{n}-u_{n}}(B)}{G(n)^{m}} \stackrel{\sqrt[222]{\leqslant}}{\leqslant} C_{r_{n}-u_{n}}(B) r_{n}^{m-s} \frac{1}{(1-1 / n)^{m}}
$$

and since $\frac{1}{(1-1 / n)^{m}} \rightarrow 1$ and, by (23),$\frac{u_{n}}{r_{n}} \rightarrow 1$, we get

$$
\mu(B) \leqslant \varlimsup_{n \rightarrow \infty} C_{r_{n}-u_{n}}(B)\left(r_{n}-u_{n}\right)^{m-s} \leqslant \overline{\boldsymbol{\nu}}_{0}^{m-s}(B) .
$$

Since this holds for every Borel set $B$, we get $\mu(E) \leqslant \overline{\boldsymbol{\nu}}^{m-s}(E)$ for every $E \subseteq \mathrm{Z}$ Borel. 
It remains to show that $\overline{\boldsymbol{\nu}}_{0}^{m-s}(Z) \leqslant 1$. Write $\theta_{n}=1+\frac{1}{n}$. Since obviously $N_{u_{n}}(\mathrm{~K})=G(n)$, we have, for all $r \in\left[u_{n}, r_{n}\right]$,

$$
N_{r}(\mathrm{~K}) r^{1-p} \leqslant N_{u_{n}}(\mathrm{~K}) r_{n}^{1-p}=G(n) r_{n}^{1-p} \stackrel{(22)}{\leqslant} \theta_{n} .
$$

Now suppose $r \in\left[r_{n+1}, u_{n}\right]$. Note first that $N_{r}(E) \leqslant \frac{\operatorname{diam} E}{r}+1$ for any set $E \subseteq \mathbb{R}$ and therefore $N_{r}(\mathrm{~K}) \leqslant N_{t}(\mathrm{~K})\left(\frac{t}{r}+1\right)$ whenever $r<t$. In particular,

$$
\begin{gathered}
N_{r}(\mathrm{~K}) \leqslant N_{u_{n}}(\mathrm{~K})\left(\frac{u_{n}}{r}+1\right) \leqslant 2 G(n) \frac{u_{n}}{r}, \\
N_{r}(\mathrm{~K}) r^{1-p} \leqslant G(n)\left(\frac{u_{n}}{r}+1\right) r^{1-p}=G(n+1) r_{n+1} r^{-p}+G(n) r^{1-p} \\
\leqslant G(n+1) r_{n+1}^{1-p}+G(n) r_{n}^{1-p}\left(\frac{u_{n}}{r_{n}}\right)^{1-p} \stackrel{\sqrt{22})}{\leqslant} \theta_{n+1}+\theta_{n}\left(\frac{u_{n}}{r_{n}}\right)^{1-p} .
\end{gathered}
$$

Since $C_{r}(\mathrm{Z}) \leqslant\left(N_{r}(\mathrm{~K})\right)^{m}$ (cf. (15) and (21)) and since $\theta_{n} \rightarrow 1$ and $\frac{u_{n}}{r_{n}} \leqslant \frac{1}{n}$, the estimates (25) and (27) give

$$
\overline{\boldsymbol{\nu}}_{0}^{m-s}(\mathbf{Z}) \leqslant \varlimsup_{r \rightarrow 0} N_{r}(\mathbf{Z}) r^{m-s} \leqslant \varlimsup_{n \rightarrow \infty}\left(\theta_{n}+\theta_{n} / n^{1-p}\right)^{m}=1 .
$$

We proceed with the proof of the theorem. It remains to estimate $\overline{\boldsymbol{\nu}}_{0}^{m}(X \times \mathrm{Z})$ from above. For $r \in\left[u_{n}, r_{n}\right]$ we employ Lemma 5.1(i).

$$
\begin{gathered}
C_{r}(X \times \mathrm{Z}) r^{m} \leqslant C_{r}(X) r^{m}\left(N_{r}(K)\right)^{m} \leqslant 2^{m} Q C_{r_{n}}(X) r_{n}^{m} G(n)^{m} \\
\leqslant 2^{m} Q C_{r_{n}}(X) r_{n}^{s}\left(G(n) r_{n}^{1-p}\right)^{m} \stackrel{m{ }^{(22)}}{\leqslant} 2^{m} Q C_{r_{n}}(X) r_{n}^{s} \theta_{n}^{m} .
\end{gathered}
$$

For $r \in\left[r_{n+1}, u_{n}\right]$ we employ the latter estimate (26).

$$
\begin{gathered}
C_{r}(X \times \mathrm{Z}) r^{m} \leqslant C_{r}(X) r^{m}\left(N_{r}(K)\right)^{m} \leqslant C_{r_{n+1}}(X) r_{n+1}^{s}\left(2 G(n) u_{n} r_{n+1}^{-p}\right)^{m} \\
\leqslant C_{r_{n+1}}(X) r_{n+1}^{s}\left(2 G(n+1) r_{n+1}^{1-p}\right)^{m} \stackrel{(22)}{\leqslant} 2^{m} \theta_{n+1}^{m} C_{r_{n+1}}(X) r_{n+1}^{s} .
\end{gathered}
$$

Since $\theta_{n} \rightarrow 1$, (28) and (29) yield

$$
\overline{\boldsymbol{\nu}}_{0}^{m}(X \times \mathbf{Z}) \leqslant \varlimsup_{n \rightarrow \infty} 2^{m} Q C_{r_{n}}(X) r_{n}^{s} \leqslant 2^{m} Q \boldsymbol{\nu}_{\Delta, 0}^{s}(X) .
$$

The simple proof of the following corollary is omitted.

Corollary 5.4. Let $0 \leqslant s \leqslant m \in \omega$. Let $X$ be a countably $m$-homogeneous metric space such that $\underline{\boldsymbol{\nu}}_{\rightarrow}^{s}(X)<\infty$. There is a compact set $\mathbf{Z} \subseteq \mathbb{R}^{m}$ such that $\overline{\boldsymbol{\nu}}^{m-s}(\mathbf{Z})=1$ and $\overline{\boldsymbol{\nu}}^{m}(X \times \mathrm{Z})$ is $\sigma$-finite.

Theorem 5.5. Let $X$ be a metric space $X$ and $\operatorname{dim}_{\sigma \mathrm{A}} X \leqslant m \in \omega$. There is a

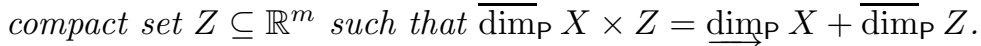

Proof. Fix $s>\operatorname{dim}_{P} X$. By Proposition 4.3 there is a scale $\Delta$ such that $\boldsymbol{\nu}_{\Delta}^{s}(X)=0$. Let $Z_{s}$ be the space $Z$ of Theorem 5.2 .

Let $\varepsilon>0$. There is a cover $X=\bigcup_{i \in \omega} X_{i}$ such that for each $i \in \omega$ there is $Q_{i}$ such that $X_{i}$ is $\left(Q_{i}, m+\varepsilon\right)$-homogeneous and moreover $\boldsymbol{\nu}_{\Delta, 0}^{s}\left(X_{i}\right)<1$. Inspect the estimates (28) and (29): If we use $\left(Q_{i}, m+\varepsilon\right)$-homogeneity of $X_{i}$ instead of $(Q, m)$-homogeneity of $X$, we get for $r \in\left[u_{n}, r_{n}\right]$

$$
C_{r}\left(X_{i} \times \mathrm{Z}_{s}\right) r^{m+\varepsilon} \leqslant 2^{m+\varepsilon} Q_{i} \theta_{n}^{m} C_{r_{n}}\left(X_{i}\right) r_{n}^{s} r_{n}^{\varepsilon}
$$


and since (29) does not depend on homogeneity of $X$, we get for $r \in\left[r_{n+1}, u_{n}\right]$

$$
C_{r}\left(X_{i} \times \mathrm{Z}_{s}\right) r^{m+\varepsilon} \leqslant C_{r}\left(X_{i} \times \mathrm{Z}_{s}\right) r^{m} r^{\varepsilon} \leqslant 2^{m} \theta_{n+1}^{m} C_{r_{n+1}}\left(X_{i}\right) r_{n+1}^{s} u_{n}^{\varepsilon} .
$$

Since $\theta_{n} \rightarrow 0, r_{n} \rightarrow 0, u_{n} \rightarrow 0$ and $\varlimsup_{n \rightarrow \infty} C_{r_{n}}\left(X_{i}\right) r_{n}^{s} \leqslant \nu_{\Delta, 0}^{s}\left(X_{i}\right)<1$, these estimates yield $\overline{\boldsymbol{\nu}}_{0}^{m+\varepsilon}\left(X_{i} \times \mathrm{Z}_{s}\right)=0$ for all $i$. Consequently $\overline{\boldsymbol{\nu}}^{m+\varepsilon}\left(X \times \mathrm{Z}_{s}\right)=0$ for all $\varepsilon>0$, whence $\overline{\operatorname{dim}}_{\mathrm{P}} X \times \mathrm{Z}_{s} \leqslant m$.

Now let $s_{k}=\operatorname{dim}_{P} X+\frac{1}{k}$. Consider the spaces $Z_{s_{k}}$. Mutatis mutandis we may assume $Z_{s_{k}} \subseteq\left[2^{-k-1}, 2^{-k}\right]$. Let $Z=\bigcup_{k \in \omega} Z_{s_{k}} \cup\{0\}$. It is clearly compact and $\overline{\operatorname{dim}}_{\mathrm{P}} X \times Z \leqslant \sup \overline{\operatorname{dim}}_{\mathrm{p}} X \times Z_{s_{k}} \leqslant m$. On the other hand, $\overline{\operatorname{dim}}_{\mathrm{p}} Z \geqslant \sup m-s_{k}=$ $m-{\underset{\operatorname{dim}}{\mathrm{P}}} X$. Thus $\overline{\operatorname{dim}}_{\mathrm{P}} X \times Z \leqslant \underline{\operatorname{dim}}_{\mathrm{P}} X+\overline{\operatorname{dim}}_{\mathrm{P}} Z$. The opposite inequality follows from Theorem 4.5 .

In particular, if $X \subseteq \mathbb{R}^{m}$, the above theorem yields a solution to the problem of $\mathrm{Hu}$ and Taylor:

Corollary 5.6. For every set $X \subseteq \mathbb{R}^{m}$ there is a compact set $Z \subseteq \mathbb{R}^{m}$ such that $\overline{\operatorname{dim}}_{\mathrm{P}} X \times \mathbf{Z}-\overline{\operatorname{dim}}_{\mathrm{P}} \mathbf{Z}=\underline{\operatorname{dim}}_{\mathrm{P}} X$. In particular, $\operatorname{aDim} X=\operatorname{dim}_{\mathrm{P}} X$.

\section{Comments And questions}

Other packing measures. The packings we used are sometimes called weak packings or pseudo-packings. There are other kinds of packing in use. The most common that we shall call true packing is this: $\left\{\left(x_{i}, r_{i}\right): i \in I\right\}$ is a true packing if the balls $B\left(x_{i}, r_{i}\right), B\left(x_{j}, r_{j}\right)$ are disjoint for distinct $i, j \in I$. There are also open balls variants. Some definitions of packing measures are based on diameters of the underlying balls, instead of radii. Various packing measures and their relations are discussed in detail e.g. in [5, 6, 4,. Analysis shows that our results are to some extent valid also for other packing measures.

Hausdorff measures. We intentionally neglected results involving Hausdorff measure and dimension. The reason is that for any Hausdorff function $h$ we have $\mathscr{H}^{h_{2}} \leqslant \underline{\boldsymbol{\nu}}^{h}$, where $h_{2}(r)=h(r / 2)$ and $\mathscr{H}^{h_{2}}$ is the corresponding Hausdorff measure. (Hint: If $\left\{\left(x_{i}, \delta\right)\right\}$ is a maximal uniform packing, then $\left\{B\left(x_{i}, \delta\right)\right\}$ is a $2 \delta$-cover.) Thus e.g. Howroyd's [11, Theorem 13] stating that $\overline{\mathscr{P}}^{g h}(X \times Y) \geqslant \overline{\mathscr{P}}^{g}(X) \mathscr{H}^{h_{2}}(Y)$ follows at once from Corollary 3.6 .

Upper estimates of cartesian products measures. All inequalities of Section 3 estimate the measures on a product by means of measures on coordinate spaces from below. We paid no attention to reverse estimates. Basic results in this direction are due to Tricot [18] and Howroyd [11, see also [5]. Howroyd has the following: Let $\overline{\mathrm{P}}^{g}$ denote the upper packing measure obtained from true packings. Let $g, h$ be right-continuous Hausdorff functions. Then $\overline{\mathrm{P}}^{g h}(X \times Y) \leqslant \overline{\mathrm{P}}^{g}(X) \overline{\mathscr{P}}^{h}(Y)$ for any metric spaces $X, Y$, as long as the product on the right is not $0 \cdot \infty$ or $\infty \cdot 0$. Inspection of the proof shows that restricting the admissible radii to a given scale does not matter. One can thus conclude that, under the same conditions and with the obvious definitions, $\overline{\mathrm{P}}_{\Delta}^{g h}(X \times Y) \leqslant \overline{\mathrm{P}}_{\Delta}^{g}(X) \overline{\mathscr{P}}_{\Delta}^{h}(Y)$ for any scale $\Delta$ and also $\underline{\mathrm{P}}^{g h}(X \times Y) \leqslant \underline{\mathrm{P}}^{g}(X) \overline{\mathscr{P}}^{h}(Y)$ and $\underline{\mathrm{P}}^{g h}(X \times Y) \leqslant \underline{\mathrm{P}}^{g}(X) \overline{\mathscr{P}}^{h}(Y)$.

Corresponding inequalities for the box measures can be derived from (20). Corresponding inequalities for dimensions (due to Tricot [18]) are well-known (except 
$\left.\operatorname{dim}_{\mathrm{P}} X \times Y \leqslant \operatorname{dim}_{\mathrm{P}} X+\overline{\operatorname{dim}}_{\mathrm{P}} Y\right)$. Combining Tricot's inequalities with the one just mentioned and Corollary 4.5 we thus have:

Theorem 6.1. For any metric spaces $X, Y$

$$
\begin{aligned}
& \underline{\operatorname{dim}}_{\mathrm{P}} X+\underline{\operatorname{dim}}_{\mathrm{P}} Y \leqslant \underline{\operatorname{dim}}_{\mathrm{P}} X \times Y \leqslant \underline{\operatorname{dim}}_{\mathrm{P}} X+\overline{\operatorname{dim}}_{\mathrm{P}} Y \text {, } \\
& {\underset{\operatorname{dim}}{P}}_{P} X+{\underset{\operatorname{dim}}{\mathrm{P}}} Y \leqslant{\underset{\operatorname{dim}}{\mathrm{P}}} X \times Y \leqslant{\underset{\operatorname{dim}}{\mathrm{P}}} X+\overline{\operatorname{dim}}_{\mathrm{P}} Y \\
& \leqslant \overline{\operatorname{dim}}_{\mathrm{P}} X \times Y \leqslant \overline{\operatorname{dim}}_{\mathrm{P}} X+\overline{\operatorname{dim}}_{\mathrm{P}} Y .
\end{aligned}
$$

Comparison of lower packing and box measures. As is obvious from Section 2, the measures $\underline{P}^{h}$ and $\underline{\nu}^{h}$ are closely related, much closer than their upper counterparts, but we do not really know much about their relation. We even do not know if they are equal. The following problems seem interesting.

Question 6.2. Is there a (compact) set $X \subseteq \mathbb{R}$ and $s>0$ such that

(i) $\underline{\nu}^{s}(X)<\mathscr{P}^{s}(X)$ ?

(ii) $\underline{\boldsymbol{\nu}}^{s}(X)=0$ and $\underline{\mathscr{P}}^{s}(X)=\infty$ ?

(iii) $\underline{\boldsymbol{\nu}}^{s}(X)=0$ and $0<\underline{\mathscr{P}}^{s}(X)<\infty$ ?

A related problem, perhaps the most interesting one, is whether one can replace $\underline{\boldsymbol{\nu}}^{g}$ with $\underline{\mathscr{P}}^{g}$ in the integrands in Theorem 3.5

Question 6.3. Is it true that inequalities in Theorem 3.5 improve to $\overline{\mathscr{P}}^{g h}(E) \geqslant$ $\int^{*} \underline{\mathscr{P}}^{g}\left(E_{x}\right) \mathrm{d} \overline{\mathscr{P}}^{h}, \underline{\mathscr{P}}^{g h}(E) \geqslant \int^{*} \underline{\mathscr{P}}^{g}\left(E_{x}\right) \mathrm{d} \underline{\mathscr{P}}^{h}$ and $\underline{\mathscr{P}}^{g h}(E) \geqslant \int^{*} \underline{\mathscr{P}}^{g}\left(E_{x}\right) \mathrm{d} \underline{\mathscr{P}}^{h} ?$

A modest variation of this problem:

Question 6.4. Let $X, Y$ be metric spaces and $g, h$ Hausdorff functions.

(i) Suppose $\mathscr{\mathscr { P }}^{g}(X)>0$ and $\overline{\mathscr{P}}^{h}(Y)>0$. Does it follow that $\overline{\mathscr{P}}^{g h}(X \times Y)>0$ ?

(ii) Suppose $\underline{\mathscr{P}}^{g}(X)>0$ and $\underline{\mathscr{P}}^{h}(Y)>0$. Does it follow that $\underline{\mathscr{P}}^{g h}(X \times Y)>0$ ?

Another interesting problem is that of semifiniteness of the $\underline{\mathscr{P}}^{h}$ and $\underline{\boldsymbol{\nu}}^{h}$. Recall that a Borel measure is semifinite if every Borel set of infinite measure contains a Borel subset of finite positive measure. By a theorem of H. Joyce and D. Preiss [12] the upper packing measure $\overline{\mathscr{P}}^{h}$ on an analytic metric space is semifinite.

Question 6.5. (i) Under what conditions imposed on $X$ and $g$ are the measures $\mathscr{P}^{g}$ and $\underline{\boldsymbol{\nu}}^{g}$ on $X$ semifinite?

(ii) Is there $s>0$ such that $\underline{\mathscr{P}}^{s}$ is not semifinite on $\mathbb{R}$ ?

Directed pre-measures. We also do not know if $\underline{\boldsymbol{\nu}}_{\rightarrow}^{g}$ and $\mathscr{P}_{\rightarrow}^{g}$ may differ.

Question 6.6. Is there a (compact) set $X \subseteq \mathbb{R}$ and $s>0$ such that

(i) $\underline{\boldsymbol{\nu}}^{s}(X)<\mathscr{P P}^{s}(X)$ ?

(ii) $\underline{\boldsymbol{\nu}}^{s}(X)=0$ and $\mathscr{\mathscr { P }}^{s}(X)=\infty$ ?

(iii) $\underline{\boldsymbol{\nu}}^{s}(X)=0$ and $0<\mathscr{P}^{s}(X)<\infty$ ?

Finite Assouad dimension hypothesis. Proofs of Section 5 inevitably depend on the finite Assouad dimension of the metric space under consideration, but there is no clue that this hypothesis is not superfluous.

Question 6.7. Is there a metric space $X$ such that $\overline{\operatorname{dim} \mathrm{P}} X<\infty$ and

$$
{\underset{\operatorname{dim}}{\mathrm{P}}}_{\mathrm{P}} X<\inf \left\{\overline{\operatorname{dim}}_{\mathrm{P}} X \times Z-\overline{\operatorname{dim}}_{\mathrm{P}} Z: \overline{\operatorname{dim}}_{\mathrm{P}} Z<\infty\right\} ?
$$


Dimensions of Borel measures. Our dimension inequalities have counterparts for dimensions of finite Borel measures. Recall that if $\mu$ is a finite Borel measure in a metric space $X$ and dim is any of the fractal dimension under consideration, the corresponding dimensions of $\mu$ are defined by

$$
\operatorname{dim} \mu=\inf \{\operatorname{dim} E: B \subseteq X \text { Borel, } \mu(E)>0\} .
$$

It is easy to check that $\underline{\operatorname{dim}}_{\mathrm{P}} \mu=\underline{\operatorname{dim}}_{\mathrm{P}} \mu=\underline{\operatorname{dim}}_{\mathrm{B}} \mu$ and $\overline{\operatorname{dim}}_{\mathrm{P}} \mu=\overline{\operatorname{dim}}_{\mathrm{B}} \mu$. Another equivalent definition of the two dimensions is $\underline{\operatorname{dim}}_{\mathrm{P}} \mu=\sup \left\{s: \mu \ll \underline{P}^{s}\right\}=\sup \{s$ : $\left.\mu \ll \underline{\boldsymbol{\nu}}^{s}\right\}$ and $\overline{\operatorname{dim}}_{\mathrm{P}} \mu=\sup \left\{s: \mu \ll \overline{\mathscr{P}}^{s}\right\}=\sup \left\{s: \mu \ll \overline{\boldsymbol{\nu}}^{s}\right\}$, where $\ll$ denotes absolute continuity.

The following theorem is a straightforward consequence of Corollary 4.5 .

Theorem 6.8. Let $\mu, \nu$ be finite Borel measures in metric spaces.

(i) $\overline{\operatorname{dim}}_{\mathrm{P}} \mu \times \nu \geqslant \overline{\operatorname{dim}}_{\mathrm{P}} \mu+\underline{\operatorname{dim}}_{\mathrm{P}} \nu$,

(ii) $\underline{\operatorname{dim}}_{P} \mu \times \nu \geqslant \underline{\operatorname{dim}}_{P} \mu+\underline{\operatorname{dim}}_{P} \nu$.

There is also a measure counterpart to Theorem 5.5 .

Theorem 6.9. Let $\mu$ be a finite Borel measure in a metric space $X$. If $\operatorname{dim}_{\sigma \mathrm{A}} X \leqslant$ $m \in \omega$, then

$$
\inf \left\{\overline{\operatorname{dim}}_{\mathrm{P}} \mu \times \nu-\overline{\operatorname{dim}}_{\mathrm{P}} \nu: \nu \text { is a finite Borel measure in } \mathbb{R}^{m}\right\}=\underline{\operatorname{dim}}_{\mathrm{P}} \mu .
$$

Proof in outline. Let $\varepsilon>0$ and $s=\underline{\operatorname{dim}}_{\mathrm{P}} \mu+\varepsilon$. There is a set $E \subseteq X$ such that $\mu(E)>0$ and $\underset{\operatorname{dim}_{P}}{\longrightarrow}<s$. By Theorem 5.5 and its proof there is a compact set $\mathrm{Z}_{s} \subseteq \mathbb{R}^{m}$ such that $\overline{\operatorname{dim}}_{\mathrm{P}} E \times \mathrm{Z}_{s} \leqslant m$ and, by Lemma [5.3, the corresponding

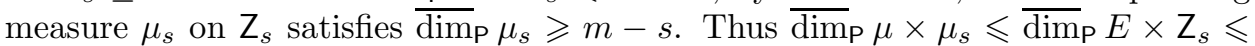
$m \leqslant \overline{\operatorname{dim}}_{\mathrm{P}} \mu_{s}+\underline{\operatorname{dim}}_{\mathrm{P}} \mu+\varepsilon$.

\section{REFERENCES}

1. Patrice Assouad, Étude d'une dimension métrique liée à la possibilité de plongements dans $\mathbf{R}^{n}$, C. R. Acad. Sci. Paris Sér. A-B 288 (1979), no. 15, A731-A734. MR 532401 (80f:54030)

2. Christopher J. Bishop and Yuval Peres, Packing dimension and Cartesian products, Trans. Amer. Math. Soc. 348 (1996), no. 11, 4433-4445. MR 1376540 (97c:28015)

3. Der-Chen Chang and Yong $\mathrm{Xu}$, New inequalities on fractal analysis and their applications, J. Inequal. Appl. (2007), Art. ID 26249, 17. MR 2291648 (2008e:28017)

4. Manav Das, Equality of the packing and pseudo-packing measures, Bull. Polish Acad. Sci. Math. 49 (2001), no. 1, 73-79. MR 1824158 (2002b:28005)

5. G. A. Edgar, Packing measure in general metric space, Real Anal. Exchange 26 (2000/01), no. 2, 831-852. MR 1844397 (2002e:28011)

6. _ Centered densities and fractal measures, New York J. Math. 13 (2007), 33-87 (electronic). MR 2288081 (2008b:28006)

7. Gerald A. Edgar, Integral, probability, and fractal measures, Springer-Verlag, New York, 1998. MR 1484412 (99c:28024)

8. Hermann Haase, A contribution to measure and dimension of metric spaces, Math. Nachr. 124 (1985), 45-55. MR 827889 (87g:54079)

9. - Open-invariant measures and the covering number of sets, Math. Nachr. 134 (1987), 295-307. MR 918685 (89c:28016)

10. Edwin Hewitt and Karl Stromberg, Real and abstract analysis. A modern treatment of the theory of functions of a real variable, Springer-Verlag, New York, 1965. MR 0188387 (32 \#5826)

11. J. D. Howroyd, On Hausdorff and packing dimension of product spaces, Math. Proc. Cambridge Philos. Soc. 119 (1996), no. 4, 715-727. MR 1362951 (96j:28006)

12. H. Joyce and D. Preiss, On the existence of subsets of finite positive packing measure, Mathematika 42 (1995), no. 1, 15-24. MR 1346667 (96g:28010) 
13. D. G. Larman, A new theory of dimension, Proc. London Math. Soc. (3) 17 (1967), $178-192$. MR 0203691 (34 \#3540)

14. Jouni Luukkainen, Assouad dimension: antifractal metrization, porous sets, and homogeneous measures, J. Korean Math. Soc. 35 (1998), no. 1, 23-76. MR 1608518 (99m:54023)

15. Pertti Mattila, Geometry of sets and measures in Euclidean spaces, Cambridge Studies in Advanced Mathematics, vol. 44, Cambridge University Press, Cambridge, 1995, Fractals and rectifiability. MR 1333890

16. Yakov B. Pesin, Dimension theory in dynamical systems, Chicago Lectures in Mathematics, University of Chicago Press, Chicago, IL, 1997, Contemporary views and applications. MR 1489237 (99b:58003)

17. C. A. Rogers, Hausdorff measures, Cambridge University Press, London, 1970. MR 0281862 (43 \#7576)

18. Claude Tricot, Jr., Two definitions of fractional dimension, Math. Proc. Cambridge Philos. Soc. 91 (1982), no. 1, 57-74. MR 633256 (84d:28013)

19. Yimin Xiao, Packing dimension, Hausdorff dimension and Cartesian product sets, Math. Proc. Cambridge Philos. Soc. 120 (1996), no. 3, 535-546. MR 1388205 (97d:28006)

Department of Mathematics, Faculty of Civil Engineering, Czech Technical UniverSity, Thákurova 7, 16000 Prague 6, Czech Republic

E-mail address: zindulka@mat.fsv.cvut.cz

$U R L$ : http://mat.fsv.cvut.cz/zindulka 